\title{
One-loop Feynman integral reduction by differential operators
}

\author{
Chang $\mathrm{Hu},{ }^{1,2, \dagger}$ Tingfei $\mathrm{Li}^{1,{ }^{1, *}}$ and Xiaodi $\mathrm{Li}^{3,4, \$}$ \\ ${ }^{1}$ Zhejiang Institute of Modern Physics, Zhejiang University, \\ Hangzhou 310027, People's Republic of China \\ ${ }^{2}$ Hangzhou Institute of Advanced Study, UCAS, Hangzhou 310027, People's Republic of China \\ ${ }^{3}$ Interdisciplinary Center for Theoretical Study, University of Science and Technology of China, \\ Hefei, Anhui 230026, China \\ ${ }^{4}$ Peng Huanwu Center for Fundamental Theory, Hefei, Anhui 230026, China
}

\begin{abstract}
(Received 2 November 2021; accepted 22 November 2021; published 20 December 2021)
\end{abstract}
\begin{abstract}
For loop integrals the standard method is reduction. A well-known reduction method for one-loop integrals is the Passarino-Veltman (PV) reduction. Inspired by the recent paper [B. Feng, T. Li, and X. Li, J. High Energy Phys. 09 (2021) 081.] where the tadpole reduction coefficients have been solved, in this paper we show the same technique can be used to give a complete integral reduction for any one-loop integrals. The differential operator method is an alternative version of the PV-reduction method. Using this method, analytic expressions of all reduction coefficients of the master integrals can be given by algebraic recurrence relation easily. We demonstrate our method explicitly with several examples.
\end{abstract}

DOI: 10.1103/PhysRevD.104.116014

\section{INTRODUCTION}

The calculation of the scattering amplitude at higherloop level is always like a chronic disease to block the evolution of high energy physics. Theoretical physicists have made many prescriptions to cure this problem starting in the 1970s. The most significant solution is to reduce a loop amplitude into a linear combination of some scalar master integrals (MIs) under dimensional regularization [1-17]

$$
I^{1-\text { loop }}=\sum_{i_{d_{0}+1}} C^{i_{d_{0}+1}} I_{d_{0}+1}^{i_{d_{0}+1}}+\sum_{i_{d_{0}}} C^{i_{d_{0}}} I_{d_{0}}^{i_{d_{0}}}+\cdots+\sum_{i_{1}} C^{i_{1}} I_{1}^{i_{1}}
$$

where $i_{s}$ is the set of propagators appearing in the master integrals. The coefficient $C^{i_{s}}\left(s=1, \ldots, d_{0}+1\right)$ is simply a rational function of some Lorentz invariant such as the scalar product of external momenta, while the terms $I_{s}^{i_{s}}$ are the $s$-gon scalar integrals. With the general expansion (1.1), the computation of general one-loop amplitudes has been

\footnotetext{
*Corresponding author. tfli@zju.edu.cn

isiahalbert@126.com

*xiaodili@ustc.edu.cn

Published by the American Physical Society under the terms of the Creative Commons Attribution 4.0 International license. Further distribution of this work must maintain attribution to the author(s) and the published article's title, journal citation, and DOI. Funded by SCOAP ${ }^{3}$.
}

switched to determining those coefficients of $C^{i_{s}}$. Many tools have been invented to shovel the brambles, such as integration by parts (IBP) [18,19], PV reduction [3], Ossola-Papadopoulos-Pittau (OPP) reduction [16,20-22], unitarity cut [14,17,23-29], etc.

All these methods can be divided into two categories, i.e., the reduction at the integrand level or the integral level. For reduction at the integrand level, [16] shows how to extract the coefficients of the four-, three-, two-, and onepoint one-loop scalar integrals from the full one-loop integrand of arbitrary scattering processes in an algebraical way. For the reduction at the integral level, an efficient way is the unitarity cut method. The main idea is to compare the imaginary part of the two sides of (1.1). However, since the loop integral is well defined using the dimensional regularization, the unitarity cut method in pure $4 D$ needs to generalize to $(4-2 \epsilon)$-dimension, which has been done in $[28,30]$. Based on this generalization, the analytic expressions for reduction coefficients (except the tadpole coefficients) have been derived in a series of papers [31-35].

In our previous work [36], we reconsider the problem by introducing differential operators $\mathcal{D}$ and $\mathcal{T}$. We first introduce an auxiliary vector $R^{\mu}$ and reduce it to master integrals, then apply differential operators to the integrals with respect to $R$. By comparing two sides of the expansion, we will achieve the recursion relations of the coefficients of the master integrals in differential form. With the knowledge of the algebraical structure of the reduction coefficients, we transform those differential equation form relations into algebraical form. In [36], we solved the remaining unsolved tadpole coefficients using this method. In this paper we will provide a general 
algorithm for calculating all reduction coefficients for a general tensor one-loop integral and give the explicit analytic results.

In Sec. II we review the derivation of the differential equations of reduction coefficients and show how to obtain recursion relations of the expansion coefficients. In Sec. III, we will solve the recursion relations of the reduction coefficients in the general case. In Sec. IV, we provide some examples and summarize the algorithm for calculating the reduction of a general tensor one-loop Feynman integral. In Appendix, we list all reduction coefficients for tensor triangles, boxes, and pentagons with rank 1 and rank 2.

\section{DIFFERENTIAL EQUATIONS AND RECURSION RELATIONS}

We will review the differential operators in [36] and show how we obtain the recursion relations of every reduction coefficient. Starting with the following general one-loop $m$-rank tensor integral with $n+1$ propagators

$$
I_{n+1}^{\mu_{1} \cdots \mu_{m}}=\int \frac{d^{D} \ell}{(2 \pi)^{D}} \frac{\ell^{\mu_{1}} \ell^{\mu_{2}} \cdots \ell^{\mu_{m}}}{P_{0} P_{1} \cdots P_{n}},
$$

where the $i$ th propagator is $P_{i}=\left(\ell-K_{i}\right)^{2}-M_{i}^{2}$ with setting $K_{0}=0$, we introduce an auxiliary vector $R^{\mu}$ and contract $\ell$ with $R$ on (2.1) $m$ times to get

$$
\begin{aligned}
I_{n+1}^{(m)}[R] & \equiv 2^{m} I_{n+1}^{\mu_{1} \cdots \mu_{m}} R_{\mu_{1}} \cdots R_{\mu_{m}} \\
& =\int \frac{d^{D} \ell}{(2 \pi)^{D}} \frac{(2 \ell \cdot R)^{m}}{P_{0} P_{1} \cdots P_{n}} .
\end{aligned}
$$

The vector $R$ lies in the same dimension as $\ell$. Equation (2.2) contains all the information in (2.1) but with a much simpler organization of tensor structure. In this paper, we will focus on $D=(4-2 \epsilon)$-dimensional space, although our method can obviously be applied to an arbitrary dimension. With this assumption, the integral $I_{n+1}^{(m)}[R]$ is reduced to the linear combination of pentagon, box, triangle, bubble, and tadpole master integrals

$$
\begin{aligned}
I_{n+1}^{(m)}[R]= & \sum_{a_{1}, a_{2}, \ldots, a_{5}} C^{\left(a_{1}, a_{2}, \ldots, a_{5}\right)}(m \mid n) I_{5}\left[a_{1}, a_{2}, \ldots, a_{5}\right] \\
& +\sum_{a_{1}, a_{2}, a_{3}, a_{4}} C^{\left(a_{1}, a_{2}, a_{3}, a_{4}\right)}(m \mid n) I_{4}\left[a_{1}, a_{2}, a_{3}, a_{4}\right]+\cdots \\
& +\sum_{a_{1}} C^{\left(a_{1}\right)}(m \mid n) I_{1}\left[a_{1}\right] .
\end{aligned}
$$

The reduction coefficients $C^{\left(a_{1}, \ldots, a_{r}\right)}(m \mid n), 1 \leq r \leq 5$ are the rational functions of external momenta $K_{i}$, masses $M_{i}$, and vector $R$. The summation in (2.3) covers all possible combinations of $r$ propagators $\left\{P_{a_{1}},,, P_{a_{r}}\right\} \subseteq$ $\left\{P_{0}, P_{1}, \ldots, P_{n}\right\}$. We will use the abbreviation $C^{i_{r}}(m \mid n)$ instead of $C^{\left(a_{1}, \ldots, a_{r}\right)}(m \mid n)$ with index set $i_{r}=\left\{a_{1}, \ldots, a_{r}\right\} \subseteq$ $\{0,1,2, \ldots, n\}$. It is easy to see vector $R$ only appears in the numerator of $C^{i_{r}}$ with the form $R \cdot R$ or $R \cdot K_{i}$, $i=1,2, \ldots, n$. We introduce the following two operators

$\mathcal{D}_{i} \equiv K_{i} \cdot \frac{\partial}{\partial R}, \quad i=1, \ldots, n ; \quad \mathcal{T} \equiv \eta^{\mu \nu} \frac{\partial}{\partial R^{\mu}} \frac{\partial}{\partial R^{\nu}}$.

We take the derivative of both sides of (2.3) by these two operators. The left-hand side will be

$$
\begin{aligned}
& \mathcal{D}_{i} I_{n+1}^{(m)}[R]=m I_{n+1 ; \hat{0}}^{(m-1)}-m I_{n+1 ; \hat{i}}^{(m-1)}+m f_{i} I_{n+1}^{(m-1)}, \\
& \mathcal{T} I_{n+1}^{(m)}[R]=4 m(m-1) M_{0}^{2} I_{n+1}^{(m-2)}+4 m(m-1) I_{n+1 ; \hat{0}}^{(m-2)},
\end{aligned}
$$

where the constant $f_{i} \equiv M_{0}^{2}+K_{i}^{2}-M_{i}^{2}$, and

$$
I_{n+1 ; \hat{i}}^{(m-1)}[R]=\int \frac{d^{D} \ell}{(2 \pi)^{D}} \frac{(2 \ell \cdot R)^{m-1}}{P_{0} P_{1} \cdots P_{i-1} P_{i+1} \cdots P_{n}},
$$

i.e., the $i$ th propagator has been removed. For the righthand side of (2.3), since the master integrals contains no $R$, the operators will act directly on coefficients $C^{i_{r}}(m \mid n)$. Therefore, we have the following equations,

$$
\begin{aligned}
\sum_{s=1}^{5} & \sum_{i_{s}}\left(\mathcal{D}_{i} C^{i_{s}}(m \mid n)\right) I_{s}^{i_{s}} \\
= & m \sum_{s=1}^{5} \sum_{i_{s}}\left(C^{i_{s}}(m-1 \mid n ; \hat{0})-C^{i_{s}}(m-1 \mid n ; \hat{i})\right. \\
& \left.+f_{i} C^{i_{s}}(m-1 \mid n)\right) I_{s}^{i_{s}},
\end{aligned}
$$

and

$$
\begin{aligned}
& \sum_{s=1}^{5} \sum_{i_{s}}\left(\mathcal{T} C^{i_{s}}(m \mid n)\right) I_{s}^{i_{s}} \\
& =4 m(m-1) \sum_{s=1}^{5} \sum_{i_{s}}\left(C^{i_{s}}(m-2 \mid n ; \hat{0})+M_{0}^{2} C^{i_{s}}(m-2 \mid n)\right) I_{s}^{i_{s}},
\end{aligned}
$$

where $C^{i_{s}}(m-1 ; \hat{i})$ is the coefficient of the master integrals $I_{s+1}^{i_{s}}$ in the reduced expansion of the tensor integral $I_{n+1 ; \hat{i}}^{(m-1)}[R]$. Assuming that all the reduction coefficients of the tensor integral $I_{n^{\prime}+1}^{\left(m^{\prime}\right)}$ with either $m^{\prime}<m$ or $n^{\prime}<n$ are known already, we can get a series of differential equations of $C^{i_{r}}(m \mid n)$ by comparing the coefficients of each master integral of both sides of (2.7) and (2.8). Without loss of generality, we choose $i_{r}=(0,1, \ldots, r) .{ }^{1}$ Then we have

\footnotetext{
${ }^{1}$ For simplicity, we will consider the reduction coefficients of master integrals with propagator $P_{0}$. Other cases can be obtained either by permutations or by momentum shifting. The details are shown in Sec. III B.
} 


$$
\begin{aligned}
& \mathcal{T} C^{(0,1, \ldots, r)}(m \mid n) \\
& =4 m(m-1) M_{0}^{2} C^{(0,1, \ldots, r)}(m-2 \mid n),
\end{aligned}
$$

and

$$
\begin{aligned}
\mathcal{D}_{i} C^{(0,1, \ldots, r)}(m \mid n)= & -m C^{(0,1, \ldots, r)}(m-1 \mid n ; \hat{i}) \\
& +m f_{i} C^{(0,1, \ldots, r)}(m-1 \mid n) .
\end{aligned}
$$

In Eq. (2.10), $C^{(0,1, \ldots, r)}(m-1 \mid n ; \hat{i})$ is the reduction coefficient of the master integral $I_{r+1}^{(0,1, \ldots, r)}$. Since $\hat{i}$ means the propagator $P_{i}$ has been removed, $C^{(0,1, \ldots, r)}(m-1 \mid n ; \hat{i})$ is zero when $i \leq r$.

Similar to the idea used in [36], we do not solve the differential equations directly, but expand the reduction coefficients according to its tensor structure

$$
\begin{aligned}
& C^{(0,1, \ldots, r)}(m \mid n) \\
& =\sum_{2 a_{0}+\sum_{k=1}^{n} a_{k}=m}\left\{c_{a_{0}, a_{1}, \ldots, a_{n}}^{(0,1, \ldots, r)}(m)\left(M_{0}^{2}\right)^{a_{0}+r-n} \prod_{k=0}^{n} s_{0 k}^{a_{k}}\right\},
\end{aligned}
$$

where the notation $s_{00} \equiv(R \cdot R), s_{0 i} \equiv\left(R \cdot K_{i}\right)$. The summation condition $2 a_{0}+\sum_{k=1}^{n} a_{k}=m$ guarantees vector $R$ appears $m$ times. The exponent of $M_{0}^{2}$ makes $c_{a_{0}, a_{1}, \ldots, a_{n}}^{(0,1, \ldots, r)}(m)$ dimensionless. The expansion coefficients $c_{a_{0}, a_{1}, \ldots, a_{n}}^{(0,1, \ldots, r)}(m)^{2}$ can only be a rational function of $\left(K_{i} \cdot K_{j}\right), i, j \neq 0$ and $M_{i}^{2},(i=0,1, \ldots, n)$. Moreover, $c_{a_{0}, a_{1}, \ldots, a_{n}}^{(0,1, \ldots)}(m)$ vanish if any $a_{k}<0, k=0,1, \ldots, n$.

For $C^{(0,1, \ldots, r)}(m-1 \mid n ; \hat{i})$ in $(2.10)$, the expansion is

$$
\begin{aligned}
C^{(0,1, \ldots, r)}(m-1 \mid n ; \hat{i}) & =\sum_{2 a_{0}+\sum_{k=1, k \neq i}^{n} a_{k}=m-1} c_{a_{0}, \ldots, a_{i-1}, a_{i+1}, \ldots, a_{n}}^{(0,1, \ldots, r)}(m-1 ; \hat{i})\left(M_{0}^{2}\right)^{a_{0}+r-n} \prod_{k=0, k \neq i}^{n} s_{0 k}^{a_{k}} \\
& =\sum_{2 a_{0}+\sum_{k=1}^{n} a_{k}=m-1} \delta_{0 a_{i}} c_{a_{0}, \ldots, a_{i}, \ldots, a_{n}}^{(0,1, \ldots, r)}(m-1 ; \hat{i})\left(M_{0}^{2}\right)^{a_{0}+r-n} \prod_{k=0}^{n} s_{0 k}^{a_{k}} .
\end{aligned}
$$

The absence of term $s_{0 i}=\left(R \cdot K_{i}\right)$ is because the propagator $P_{i}$ has been removed. In the last line of $(2.12)$, we add a factor $\delta_{0 a_{i}}$ to make the expression simpler. The subscript $\hat{a}_{i}$ means index $a_{i}$ is absent.

To get the algebraic recursion relation for expansion coefficients, we need rewrite $\mathcal{D}_{i}$ and $\mathcal{T}$ in terms of

$$
\begin{aligned}
\mathcal{D}_{i} & =K_{i}^{\mu} \frac{\partial}{\partial R^{\mu}}=2 s_{0 i} \frac{\partial}{\partial s_{00}}+\sum_{j=1}^{n} s_{i j} \frac{\partial}{\partial s_{0 j}} \\
\mathcal{T} & =2 D \frac{\partial}{\partial s_{00}}+4 s_{00} \frac{\partial^{2}}{\partial s_{00}^{2}}+4 \sum_{i=1}^{n} s_{0 i} \frac{\partial}{\partial s_{0 i}} \frac{\partial}{\partial s_{00}}+\sum_{i=1}^{n} \sum_{j=1}^{n} s_{i j} \frac{\partial}{\partial s_{0 i}} \frac{\partial}{\partial s_{0 j}} .
\end{aligned}
$$

With the above explanation, putting (2.11) and (2.12) into (2.9) and (2.10), and comparing the expansion coefficients of $\prod_{k=0}^{n} s_{0 k}^{a_{k}}$, the two types of differential equations (2.9) and (2.10) become

$$
\begin{aligned}
& \left(m+1-\sum_{l=1}^{n} i_{l}\right) c_{a_{1}, \ldots, a_{i}-1, \ldots, a_{n}}^{(0,1, \ldots, r)}(m)+\sum_{l=1}^{n}\left(a_{l}+1\right) \beta_{i l} c_{a_{1}, \ldots, a_{l}+1, \ldots, a_{n}}^{(0,1, \ldots, r)}(m) \\
& =m \alpha_{j} c_{a_{1}, \ldots, a_{n}}^{(0,1, \ldots, r)}(m-1)-m \delta_{0 a_{i}} c_{a_{1}, \ldots, a_{i}, \ldots, a_{n}}^{(0,1, \ldots, r)}(m-1 ; \hat{i}),
\end{aligned}
$$

for the $\mathcal{D}$-type and

$$
\begin{aligned}
4 m(m-1) c_{a_{1}, \ldots, a_{n}}^{(0,1, \ldots, r)}(m-2)= & \left(m-\sum_{k=1}^{n} a_{k}\right)\left(D+m+\sum_{k=1}^{n} a_{k}-2\right) c_{a_{1}, \ldots, a_{n}}^{(0,1, \ldots, r)}(m) \\
& +\sum_{0<i<j} 2\left(a_{i}+1\right)\left(a_{j}+1\right) \beta_{i j} c_{a_{1}, \ldots, a_{i}+1, \ldots, a_{j}+1, \ldots, a_{n}}^{(0,1, \ldots, r)}(m) \\
& +\sum_{i=1}^{n}\left(a_{i}+1\right)\left(a_{i}+2\right) \beta_{i i} c_{a_{1}, \ldots, a_{i}+2, \ldots, a_{n}}^{(0,1, \ldots),}(m),
\end{aligned}
$$

\footnotetext{
${ }^{2}$ Note that we use capital $C$ to represent reduction coefficient while use lower case letter $c$ to represent expansion coefficient.
} 
for the $\mathcal{T}$-type where $\alpha_{i} \equiv f_{i} / M_{0}^{2}, \beta_{i l} \equiv s_{i l} / M_{0}^{2}$ for simplicity. Again we need to emphasize $c_{a_{1}, \ldots, \hat{a}_{i}, \ldots, i_{n}}^{(0,1, \ldots)}(m-1 ; \hat{i})=0$ in the case $i \leq r$ for the same reason as discussed before. In (2.14) and (2.15) we have ignored the subscript $a_{0}$ because it has been uniquely determined by the restrictive condition $2 a_{0}+\sum_{k=1}^{n} a_{n}=m$ in (2.11).

\section{ALGORITHM FOR RECURSION RELATIONS}

The recurrence relations (2.14) and (2.15) are the key relations throughout the whole paper. In this section we show how to solve expansion coefficients by these two relations systematically.

\section{A. Reduction coefficient of $I_{r+1}[0,1, \ldots, r]$}

First, we choose the master basis containing propagators $\left\{P_{0}, P_{1}, \cdot, P_{r}\right\}$. We start by rewriting $\mathcal{D}$-type relations (2.14) in a compact form as
$\tilde{\boldsymbol{G}} \boldsymbol{T} \boldsymbol{c}^{(0,1, \ldots, r)}\left(a_{1}, \ldots, a_{n} ; m\right)=\boldsymbol{O}^{(0,1, \ldots, r)}\left(a_{1}, \ldots, a_{n} ; m\right)$,

where $\tilde{\boldsymbol{G}}=\left[\beta_{i j}\right]$ is the $n \times n$ rescaled Gram matrix and $\boldsymbol{T}$ is a diagonal matrix

$$
\boldsymbol{T}=\operatorname{diag}\left(a_{1}+1, a_{2}+1, \ldots, a_{n}+1\right) .
$$

The $\boldsymbol{c}^{(0,1, \ldots, r)}\left(a_{1}, \ldots, a_{n} ; m\right), \boldsymbol{O}^{(0,1, \ldots, r)}\left(a_{1}, \ldots, a_{n} ; m\right)$ are two vectors defined as

$\left[\boldsymbol{c}^{(0,1, \ldots, r)}\left(a_{1}, \ldots, a_{n} ; m\right)\right]_{i}=c_{a_{1}, a_{2}, \ldots, a_{i}+1, \ldots, a_{n}}^{(0,1, \ldots, r)}(m)$,

and

$$
\begin{aligned}
{\left[\boldsymbol{O}^{(0,1, \ldots, r)}\left(a_{1}, \ldots, a_{n} ; m\right)\right]_{i}=} & m \alpha_{i} c_{a_{1}, \ldots, a_{n}}^{(0,1, \ldots)}(m-1)-m \delta_{0 a_{i}} c_{a_{1}, \ldots, a_{i}, \ldots, a_{n}}^{(0,1, \ldots, r)}(m-1 ; \hat{i}) \\
& -\left(m+1-\sum_{l=1}^{n} a_{l}\right) c_{a_{1}, \ldots, a_{i}-1, \ldots, a_{n}}^{(0,1, \ldots)}
\end{aligned}
$$

The definition of these two vectors are purposely for the recurrence construction. The vector $\boldsymbol{c}$ contains coefficients with rank $m$ and subscript with the summation $1+\sum_{i} a_{i}$, while the vector $\boldsymbol{O}$ contains coefficients of three different patterns: (1) the first term with coefficients of rank $m-1$; (2) the second one with coefficients of master integrals with one less propagator and lower rank $m-1$; (3) the third one with coefficients of same rank $m$, but the summation $-1+\sum_{i} a_{i}$ of the subscript. By induction assumption, the first two terms are considered to be known already. Thus, by rewriting (3.1) as

$$
\begin{aligned}
& \boldsymbol{c}^{(0,1, \ldots, r)}\left(a_{1}, \ldots, a_{n} ; m\right) \\
& \quad=\boldsymbol{T}^{-1} \tilde{\boldsymbol{G}}^{-1} \boldsymbol{O}^{(0,1, \ldots, r)}\left(a_{1}, \ldots, a_{n} ; m\right),
\end{aligned}
$$

we have established the recurrence relations between expansion coefficients with a higher summation of subscript and those of the same rank but with the summation of the subscript reduced by two.

Iteratively using (3.5), we have two kinds of unknown expansion coefficients left

$$
\begin{aligned}
c_{0,0, \ldots, 0}^{(0,1, \ldots, r)}(m), & m & =2 k, \\
c_{1,0, \ldots, 0}^{(0,1, \ldots, r)} ; c_{0,1, \ldots, 0}^{(0,1, \ldots, r)} ; \cdots ; c_{0,0, \ldots, 1}^{(0,1, \ldots, r)}, & m & =2 k+1,
\end{aligned}
$$

depending on the parity of $m$. For the odd case $m=2 k+1$, we solve $c_{1,0, \ldots, 0}^{(0,1, \ldots, r)}$ by (3.5) again. To see it, setting $a_{1}=\cdots=a_{n}=0$ in (3.5), the left-hand side becomes

$$
\left(c_{1,0, \ldots, 0}^{(0,1, \ldots, r)}(2 k+1), c_{0,1, \ldots, 0}^{(0,1, \ldots, r)}(2 k+1), \ldots, c_{0,0, \ldots, 1}^{(0,1, \ldots, r)}(2 k+1)\right)^{T}
$$

while the right-hand side is

$$
\boldsymbol{T}^{-1} \tilde{\boldsymbol{G}}^{-1} \boldsymbol{O}^{(0,1, \ldots, r)}(0, \ldots, 0 ; 2 k+1),
$$

where

$$
\begin{aligned}
& {\left[\boldsymbol{O}^{(0,1, \ldots, r)}(0, \ldots, 0 ; 2 k+1)\right]_{i}} \\
& \quad=m \alpha_{i} c_{0, \ldots, 0}^{(0,1, \ldots, r)}(2 k)-(2 k+1) c_{0, \ldots, \hat{a}_{i}, \ldots, 0}^{(0,1, \ldots, r)}(2 k ; \hat{i}),
\end{aligned}
$$

since the third term $c_{0, \ldots,-1, \ldots, 0}^{(0,1, \ldots, r)}(2 k+1)$ vanishes. Therefore, we have reduced it to the problem of solving $c_{0, \ldots, 0}^{(0,1, \ldots, r)}(2 k)$.

Determining the value of $c_{0,0, \ldots, 0}^{(0,1, \ldots, r)}(2 k)$ requires $\mathcal{T}$-type recursion relations. For $m=2 k$ and $a_{1}=a_{2}=\cdots=a_{n}=0$, $\mathcal{T}$-type recursion relation becomes 


$$
\begin{aligned}
8 k(2 k-1) c_{0, \ldots, 0}^{(0,1, \ldots, r)}(2 k-2)= & 2 k(D+2 k-2) c_{0, \ldots, 0}^{(0,1, \ldots, r)}(2 k)+\sum_{0<i<j<n} 2 \beta_{i j} c_{0, \ldots, 1, \ldots, 1, \ldots, 0}^{(0,1, \ldots, r)}(2 k) \\
& +\sum_{i=1}^{n} 2 \beta_{i i} c_{0, \ldots, 2, \ldots, 0}^{(0,1, \ldots, r)}(2 k) .
\end{aligned}
$$

In $c_{0, \ldots, 1, \ldots, 1, \ldots, 0}^{(0,1, \ldots, r)}(2 k)$, indices 1 appear in the both $i$ th and $j$ th positions, while in $c_{0, \ldots, 2, \ldots, 0}^{(0,1, \ldots, r)}(2 k)$ index 2 appears in the $i$ th position. For $c_{0, \ldots, 2, \ldots, 0}^{(0,1, \ldots, r)}(2 k)$ and $c_{0, \ldots, 1, \ldots, 1, \ldots, 0}^{(0,1, \ldots, r)}(2 k)$ in (3.10), we use (3.5) again to reach $c_{0, \ldots, 0}^{(0,1, \ldots, r)}(2 k)$. Then we establish the relation reduced from rank $2 k$ to $2 k-2$

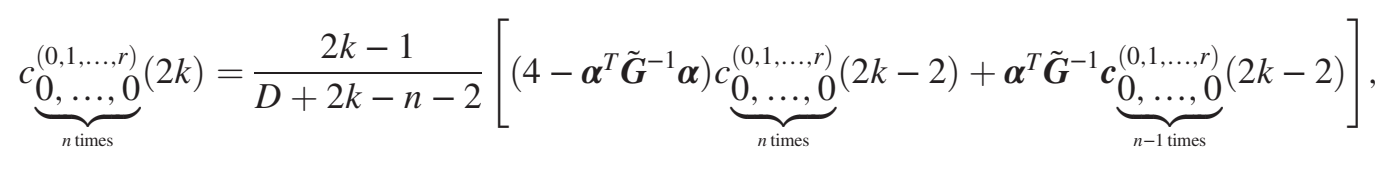

where $\boldsymbol{\alpha}$ is a vector defined as

$$
(\alpha)^{T}=\left(\alpha_{1}, \alpha_{2}, \ldots, \alpha_{n}\right)^{T}=\left(\frac{f_{1}}{M_{0}^{2}}, \frac{f_{2}}{M_{0}^{2}}, \ldots, \frac{f_{n}}{M_{0}^{2}}\right)^{T} .
$$

In the second term of right-hand side of $(3.11), \underbrace{c_{0,1, \ldots, r)}^{(0, \ldots, 0}}_{n-1 \text { times }}(m)$ is a vector defined as

$$
\begin{aligned}
& (\underbrace{c_{\underbrace{0,1, \ldots, r)}}^{0, \ldots, 0}}_{n-1 \text { times }}(m))^{T}=(\underbrace{c^{(0,1, \ldots, r)}}_{n-1 \text { times }}(m ; \hat{1}), c_{\underbrace{0, \ldots, \ldots, 0,0}_{n-1 \text { t times }}}^{(0,1, \ldots, r)}(m ; \hat{2}), \ldots, c_{\underbrace{0, \ldots, 1, \ldots, r)}_{n-1 \text { t times }}}^{(0, \ldots, 0}(m ; \hat{n}))^{T} \\
& =\left(0,0, \ldots, 0, c_{\underbrace{0} \text { t times }}^{(0,1, \ldots, r)}(m ; r \hat{+} 1), \ldots, c_{\underbrace{0, \ldots, 0,0}_{n-1 \text { times }}}^{(0,1, \ldots, r)}(m ; \hat{n})\right)^{T} .
\end{aligned}
$$

The zero of first $r$ components has been explained by Eq. (2.10). Equation (3.11) reduced rank $m$ by two. Furthermore, we see a propagator is removed in the second term of the right-hand side. Therefore, if we utilize the $\mathcal{T}$-type recursion relation repeatedly, we will end up with one of the following two cases. (1) The rank $m$ is reduced to zero, which is related to the reduction coefficient of a master integral; so it is either 1 or 0 . (2) One of the propagators $P_{i}, i \leq r$ has been removed. In this case the coefficients must be zero because the master integral will not appear in the reduction.

To make a long story short, for $d_{0}=4$, we summarize the whole reduction process below:

(a) Step 1: For a tensor integral with more than five propagators, we reduce it to a five-, four-, three-, two-, one-gon tensor integral.

(b) Step 2: For an arbitrary rank $m_{0}$, we take each $m \leq m_{0}$ arranged from small to large. If $m$ is even, we calculate the expansion coefficients $c_{a_{1}, \ldots, a_{n}}^{(0,1, \ldots, r)}(m)$ in the order $\sum_{i=1}^{n} a_{i}=0,2,4, \ldots, m$ using (3.5) and (3.10). If $m$ is odd, we calculate the expansion coefficients in the order $\sum_{i=1}^{n} a_{i}=1,3,5, \ldots, m$ using (3.5).

(c) Step 3: We continue the Step 2 until $m=m_{0}$. (d) Final step: Combining all expansion coefficients $c_{a_{1}, \ldots, a_{n}}^{(0,1, \ldots)}\left(m_{0}\right)$, we obtain the reduction coefficient by $(2.11)$.

\section{B. Calculate general $C^{\left(j_{0}, j_{1}, \ldots . j_{r}\right)}(\boldsymbol{m} \mid \boldsymbol{n})$ from $C^{(\mathbf{0}, 1, \ldots, r)}(\boldsymbol{m} \mid \boldsymbol{n})$}

In this section, we will show how to obtain the reduction coefficients of other MIs from the result of $C^{(0,1, \ldots, r)}(m \mid n)$. Let us begin with the case that the master integral contains propagators $P_{0}$. It is obvious that tensor integral $I_{n+1}^{(m)}$ is invariant under a permutation of labels $\{1,2, \ldots, n\}$. Then the reduction coefficients $C^{\left(0, j_{1} \cdots, j_{r}\right)}(m \mid n)$ is simply given by a proper replacement $\sigma:\left\{M_{i}, K_{i}\right\} \rightarrow\left\{M_{j_{i}}, K_{j_{i}}\right\},(i=1,2, \ldots, n)$,

$$
C^{\left(0, j_{1} \cdots, j_{r}\right)}(m \mid n)=\sigma C^{(0,1, \ldots, r)}(m \mid n)
$$

Now the remaining part is those MIs without $P_{0}$. Note that by a loop-momenta shift $\ell \rightarrow \ell+K_{j_{0}}$ we have 


$$
\begin{aligned}
I_{n+1}^{(m)}[R] & \rightarrow \int \frac{d^{D} \ell}{(2 \pi)^{D}} \frac{\left(2 \ell \cdot R+2 K_{j_{0}} \cdot R\right)^{m}}{\left(\ell^{2}-M_{j_{0}}^{2}\right)\left[\left(\ell+K_{j_{0}}\right)^{2}-M_{0}^{2}\right] \prod_{i=1, i \neq j_{0}}\left[\left(\ell-\left(K_{i}-K_{j_{0}}\right)^{2}-M_{i}^{2}\right)\right]} \\
& =\sum_{k=0}^{m}\left(\begin{array}{c}
m \\
k
\end{array}\right)\left(2 R \cdot K_{j_{0}}\right)^{m-k} \int \frac{d^{D} \ell}{(2 \pi)^{D}} \frac{(2 \ell \cdot R)^{k}}{\left(\ell^{2}-M_{j_{0}}^{2}\right)\left[\left(\ell+K_{j_{0}}\right)^{2}-M_{0}^{2}\right] \prod_{i=1, i \neq j_{0}}\left[\left(\ell-\left(K_{i}-K_{j_{0}}\right)^{2}-M_{i}^{2}\right)\right]} .
\end{aligned}
$$

By variable substitution $K_{j_{0}} \rightarrow-K_{j_{0}}, K_{i} \rightarrow K_{i}-K_{j_{0}}, M_{j_{0}} \leftrightarrow M_{0}$ inside the integrand, ${ }^{3}$ we arrive at the same form as (2.1). Then we have

$$
\begin{aligned}
C^{\left(j_{0}, j_{1}, \ldots, j_{r}\right)}(m \mid n) & =\sum_{k=0}^{m}\left(\begin{array}{c}
m \\
k
\end{array}\right)\left(2 R \cdot K_{j_{0}}\right)^{m-k}\left[\left.C^{\left(0, j_{1}, \ldots, j_{r}\right)}(k \mid n)\right|_{K_{j_{0}} \rightarrow-K_{j_{0}}, K_{i} \rightarrow K_{i}-K_{j_{0}}, M_{j_{0}} \leftrightarrow M_{0}}\right] \\
& =\left.\left[\sum_{k=0}^{m}\left(\begin{array}{c}
m \\
k
\end{array}\right)\left(-2 R \cdot K_{j_{0}}\right)^{m-k} C^{\left(0, j_{1}, \ldots, j_{r}\right)}(k \mid n)\right]\right|_{K_{j_{0}} \rightarrow-K_{j_{0}}, K_{i} \rightarrow K_{i}-K_{j_{0}}, M_{j_{0}} \leftrightarrow M_{0}}
\end{aligned} .
$$

\section{EXAMPLES}

Having presented the general algorithm, in this section we will use various examples to demonstrate the use of the algorithm. In the first subsection, we will show how to reduce any tensor bubble to the basis of a scalar bubble and two scalar tadpoles. The reduction of tensor triangles, tensor boxes, and tensor pentagons of rank 1 and rank 2 has been given in the Appendix. In the second subsection, we will show how to get the reduction coefficients of tensor box with rank 1 to scalar triangles without $P_{0}$ from the result of $C^{(0,1,2)}(1 \mid 3)$ using (3.16).

\section{A. The reduction of tensor bubble}

The reduction of tensor bubble $I_{2}^{(m)}$ will contain three MIs as below

Tadpoles: $I_{1}[0], I_{1}[1]$,

Bubbles: $I_{2}[0,1]$, and we have the expansion

$$
\begin{aligned}
I_{2}^{(m)}= & C^{(0)}(m \mid 1) I_{1}[0]+C^{(1)}(m \mid 1) I_{1}[1] \\
& +C^{(0,1)}(m \mid 1) I_{2}[0,1] .
\end{aligned}
$$

The way to achieve $C^{(0)}(m \mid 1)$ has been given in [36]. Here we only provide how to calculate $C^{(1)}(m \mid 1)$ and $C^{(0,1)}(m \mid 1)$. The coefficient of $I_{1}[1]$ can be obtained by (3.16) from $C^{(0)}(m \mid 1)$. While for $I_{2}[0,1]$, there is only one subscript in the expansion coefficients. So, the expansion of $C^{(0,1)}(m \mid 1)$ is

$$
C^{(0,1)}(m \mid 1)=\sum_{i} c_{i}^{(0,1)}(m)\left[M_{0}^{2}(R \cdot R)\right]^{\frac{m-i}{2}}\left(R \cdot K_{1}\right)^{i}
$$

We have the corresponding $\mathcal{D}$-type recursion relation

$$
\begin{aligned}
c_{i+2}^{(0,1)}(m) & =\frac{1}{(i+2) \beta_{11}}\left(m \alpha_{1} c_{i+1}^{(0,1)}(m-1)-m \delta_{0, i+1} c^{(0,1)}(m-1)-(m-i) c_{i}^{(0,1)}(m)\right) \\
& =\frac{1}{(i+2) \beta_{11}}\left(m \alpha_{1} c_{i+1}^{(0,1)}(m-1)-(m-i) c_{i}^{(0,1)}(m)\right),
\end{aligned}
$$

and the $\mathcal{T}$-type recursion relation

$$
\begin{aligned}
c_{0}^{(0,1)}(2 r) & =\frac{2 r-1}{2 r+D-3}\left[\left(4-\frac{\alpha_{1}^{2}}{\beta_{11}}\right) c_{0}^{(0,1)}(2 r-2)+\frac{\alpha_{1}}{\beta_{11}} c^{(0,1)}(2 r-2)\right] \\
& =\frac{2 r-1}{2 r+D-3}\left(4-\frac{\alpha_{1}^{2}}{\beta_{11}}\right) c_{0}^{(0,1)}(2 r-2),
\end{aligned}
$$

where $c^{(0,1)}(m)$ and $c^{(0,1)}(2 r-2)$ without subscripts stand for $c_{\widehat{a_{1}}}^{(0,1)}(m)$ and $c_{\widehat{a_{1}}}^{(0,1)}(2 r-2)$. These two terms vanish because they come from the reduction coefficient of bubble $I_{2}[0,1]$ for a tensor tadpole, i.e., the propagator $P_{1}$ has been removed.

\footnotetext{
${ }^{3}$ Note that we don't substitute $K_{j_{0}}$ in $\left(2 R \cdot K_{j_{0}}\right)^{m-k}$.
} 
Now we show the result for rank $m \leq 4$. The rank $m=0$ is trivial. For other ranks:

(a) $m=1$

The reduction coefficients of tadpoles: $I_{1}[0], I_{1}[1]$ Using the result in [36], we have

$$
C^{(0)}(1 \mid 1)=-\frac{R \cdot K_{1}}{K_{1}^{2}}
$$

For $I_{1}[1]$, by choosing $j_{0}=1$ in (3.16), we have

$$
\begin{aligned}
C^{(1)}(1 \mid 1) & =\left.C^{(0)}(1 \mid 1)\right|_{K_{1} \rightarrow-K_{1}, M_{0} \leftrightarrow M_{1}} \\
& =\frac{R \cdot K_{1}}{K_{1}^{2}} .
\end{aligned}
$$

The reduction coefficients of bubble: $I_{2}[0,1]$

The expansion of $C^{(0,1)}(1 \mid 1)$ becomes

$$
C^{(0,1)}(1 \mid 1)=c_{1}^{(0,1)}(1)\left(R \cdot K_{1}\right)
$$

By (4.4), we have

$$
\begin{aligned}
c_{1}^{(0,1)}(1) & =\frac{1}{\beta_{11}}\left(\alpha_{1} c_{0}^{(0,1)}(0)-2 c_{-1}^{(0,1)}(1)\right) \\
& =\frac{f_{1}}{s_{11}}
\end{aligned}
$$

where the boundary conditions are $c_{0}^{(0,1)}(0)=1$, $c_{-1}^{(0,1)}(1)=0$. Then

$$
\begin{aligned}
C^{(0,1)}(1 \mid 1) & =c_{1}^{(0,1)}(1) R \cdot K_{1} \\
& =\frac{\left(K_{1} \cdot K_{1}+M_{0}^{2}-M_{1}^{2}\right) R \cdot K_{1}}{K_{1} \cdot K_{1}} .
\end{aligned}
$$

(b) $m=2$

The reduction coefficients of tadpoles: $I_{1}[0], I_{1}[1]$

The reduction coefficient of tadpole $I_{1}[0]$ is

$$
C^{(0)}(2 \mid 1)=\frac{\left(K_{1} \cdot K_{1}+M_{0}^{2}-M_{1}^{2}\right)\left(K_{1} \cdot K_{1} R \cdot R-D\left(R \cdot K_{1}\right)^{2}\right)}{(D-1)\left(K_{1} \cdot K_{1}\right)^{2}} .
$$

For $I_{1}[1]$, by choosing $j_{0}=1$ in (3.16), we have

$$
\begin{aligned}
C^{(1)}(2 \mid 1) & =2\left(2 R \cdot K_{1}\right)\left[\left.C^{(0)}(1 \mid 1)\right|_{K_{1} \rightarrow-K_{1}, M_{0} \leftrightarrow M_{1}}\right]+\left.C^{(0)}(2 \mid 1)\right|_{K_{1} \rightarrow-K_{1}, M_{0} \leftrightarrow M_{1}} \\
& =\frac{4\left(R \cdot K_{1}\right)^{2}}{K_{1}^{2}}+\frac{\left(K_{1} \cdot K_{1}+M_{1}^{2}-M_{0}^{2}\right)\left(K_{1} \cdot K_{1} R \cdot R-D\left(R \cdot K_{1}\right)^{2}\right)}{(D-1)\left(K_{1} \cdot K_{1}\right)^{2}} .
\end{aligned}
$$

The reduction coefficients of bubble: $I_{2}[0,1]$

The expansion of $C^{(0,1)}(2 \mid 1)$ is

$$
C^{(0,1)}(2 \mid 1)=c_{0}^{(0,1)}(2) M_{0}^{2} s_{00}+c_{2}^{(0,1)}(2) s_{01}^{2} \text {. }
$$

By setting $r=1$ in (4.5), we have

$$
c_{0}^{(0,1)}(2)=\frac{1}{D-1}\left(4-\frac{\alpha_{1}^{2}}{\beta_{11}}\right) c_{0}^{(0,1)}(0)=\frac{1}{D-1}\left(4-\frac{\alpha_{1}^{2}}{\beta_{11}}\right)=\frac{4}{D-1}-\frac{f_{1}^{2}}{(D-1) M_{0}^{2} s_{11}},
$$

where the boundary condition is $c_{0}^{(0,1)}(0)=1$. By setting $i=0$ in (4.4), we have

$$
c_{2}^{(0,1)}(2)=\frac{1}{2 \beta_{11}}\left(2 \alpha_{1} c_{1}^{(0,1)}(1)-2 c_{0}^{(0,1)}(2)\right)=\frac{1}{\beta_{11}}\left(\alpha_{1} \frac{f_{1}}{s_{11}}-\frac{1}{D-1}\left(4-\frac{\alpha_{1}^{2}}{\beta_{11}}\right)\right)=\frac{D f_{1}^{2}}{(D-1) s_{11}^{2}}-\frac{4 M_{0}^{2}}{(D-1) s_{11}}
$$

where $c_{1}^{(0,1)}(1)$ has been presented in the case $m=1$. Then

$$
C^{(0,1)}(2 \mid 1)=\frac{s_{01}^{2}\left(D f_{1}^{2}-4 M_{0}^{2} s_{11}\right)+s_{00} s_{11}\left(4 M_{0}^{2} s_{11}-f_{1}^{2}\right)}{(D-1) s_{11}^{2}} .
$$


(c) $m=3$

The reduction coefficients of tadpoles: $I_{1}[0], I_{1}[1]$

The reduction coefficient of tadpole $I_{1}[0]$ is

$$
C^{(0)}(3 \mid 1)=\frac{f_{1}^{2}\left(3 s_{00} s_{01} s_{11}-(D+2) s_{01}^{3}\right)}{(D-1) s_{11}^{3}}+\frac{4 M_{0}^{2} s_{01}\left(2 s_{01}^{2}-3 s_{00} s_{11}\right)}{D s_{11}^{2}}
$$

By choosing $j_{0}=1$ in (3.16), we have

$$
\begin{aligned}
C^{(1)}(3 \mid 1)= & 3\left(2 R \cdot K_{1}\right)^{2}\left[\left.C^{(0)}(1 \mid 1)\right|_{K_{1} \rightarrow-K_{1}, M_{0} \leftrightarrow M_{1}}\right]+3\left(2 R \cdot K_{1}\right)\left[\left.C^{(0)}(2 \mid 1)\right|_{K_{1} \rightarrow-K_{1}, M_{0} \leftrightarrow M_{1}}\right] \\
& +C^{(0)}(3 \mid 1)\left[\left.C^{(0)}(3 \mid 1)\right|_{K_{1} \rightarrow-K_{1}, M_{0} \leftrightarrow M_{1}}\right] \\
= & \frac{s_{01}\left(7 D^{2} s_{01}^{2}+12 D M_{1}^{2} s_{00}-10 D s_{01}^{2}-12 M_{1}^{2} s_{00}\right)}{(D-1) D s_{11}}+\frac{(D+2)\left(M_{0}^{2}-M_{1}^{2}\right)^{2} s_{01}^{3}}{(D-1) s_{11}^{3}} \\
& +\frac{4\left(D M_{0}^{2}-D M_{1}^{2}-2 M_{1}^{2}\right) s_{01}^{3}}{D s_{11}^{2}}-\frac{3\left(M_{0}^{2}-M_{1}^{2}\right)^{2} s_{00} s_{01}}{(D-1) s_{11}^{2}}+\frac{3 s_{00} s_{01}}{D-1} .
\end{aligned}
$$

The reduction coefficients of bubble: $I_{2}[0,1]$

The expansion of $C^{(0,1)}(3 \mid 1)$ is

$$
C^{(0,1)}(3 \mid 1)=c_{1}^{(0,1)}(3) M_{0}^{2} s_{00} s_{01}+c_{3}^{(0,1)}(3) s_{01}^{3} .
$$

By setting $i=-1$ in (4.4), we have

$$
c_{1}^{(0,1)}(3)=\frac{1}{\beta_{11}}\left(3 \alpha_{1} c_{0}^{(0,1)}(2)-4 c_{-1}^{(0,1)}(3)\right)=\frac{1}{\beta_{11}}\left[3 \alpha_{1}\left(\frac{4}{D-1}-\frac{f_{1}^{2}}{(D-1) M_{0}^{2} s_{11}}\right)\right]=\frac{12 f_{1}}{(D-1) s_{11}}-\frac{3 f_{1}^{3}}{(D-1) M_{0}^{2} s_{11}^{2}},
$$

where we have used $c_{-1}^{(0,1)}(3)=0$ and the result of expansion coefficient $c_{0}^{(0,1)}(2)$ in the case $m=2$. By setting $i=1$ in (4.4), we have

$$
\begin{aligned}
c_{3}^{(0,1)}(3) & =\frac{1}{3 \beta_{11}}\left(3 \alpha_{1} c_{2}^{(0,1)}(2)-2 c_{1}^{(0,1)}(3)\right) \\
& =\frac{1}{3 \beta_{11}}\left[3 \alpha_{1}\left(\frac{D f_{1}^{2}}{(D-1) s_{11}^{2}}-\frac{4 M_{0}^{2}}{(D-1) s_{11}}\right)-2\left(\frac{12 f_{1}}{(D-1) s_{11}}-\frac{3 f_{1}^{3}}{(D-1) M_{0}^{2} s_{11}^{2}}\right)\right] \\
& =\frac{(D+2) f_{1}^{3}}{(D-1) s_{11}^{3}}-\frac{12 f_{1} M_{0}^{2}}{(D-1) s_{11}^{2}} .
\end{aligned}
$$

Then the reduction coefficient is

$$
C^{(0,1)}(3 \mid 1)=\frac{f_{1}\left(s_{01}^{3}\left((D+2) f_{1}^{2}-12 M_{0}^{2} s_{11}\right)+3 s_{00} s_{11} s_{01}\left(4 M_{0}^{2} s_{11}-f_{1}^{2}\right)\right)}{(D-1) s_{11}^{3}}
$$

(d) $m=4$

The reduction coefficients of tadpoles: $I_{1}[0], I_{1}[1]$

The reduction coefficient of tadpole $I_{1}[0]$ is 


$$
\begin{aligned}
C^{(0)}(4 \mid 1)= & -\frac{3 f_{1} s_{00}\left(8 D^{2} M_{0}^{2} s_{01}^{2}+D f_{1}^{2} s_{00}+16 D M_{0}^{2} s_{01}^{2}-16 M_{0}^{2} s_{01}^{2}\right)}{(D-1) D(D+1) s_{11}^{2}} \\
& +\frac{2(D+2) f_{1} s_{01}^{2}\left(3 D f_{1}^{2} s_{00}+10 D M_{0}^{2} s_{01}^{2}-8 M_{0}^{2} s_{01}^{2}\right)}{(D-1) D(D+1) s_{11}^{3}}+\frac{12(2 D-1) f_{1} M_{0}^{2} s_{00}^{2}}{(D-1) D(D+1) s_{11}} \\
& -\frac{(D+2)(D+4) f_{1}^{3} s_{01}^{4}}{(D-1)(D+1) s_{11}^{4}} .
\end{aligned}
$$

By choosing $j_{0}=1$ in (3.16), we have

$$
\begin{aligned}
C^{(1)}(4 \mid 1)= & 4\left(2 R \cdot K_{1}\right)^{3}\left[\left.C^{(0)}(1 \mid 1)\right|_{K_{1} \rightarrow-K_{1}, M_{0} \leftrightarrow M_{1}}\right]+6\left(2 R \cdot K_{1}\right)^{2}\left[\left.C^{(0)}(2 \mid 1)\right|_{K_{1} \rightarrow-K_{1}, M_{0} \leftrightarrow M_{1}}\right] \\
& +4\left(2 R \cdot K_{1}\right)\left[\left.C^{(0)}(3 \mid 1)\right|_{K_{1} \rightarrow-K_{1}, M_{0} \leftrightarrow M_{1}}\right]+\left[\left.C^{(0)}(4 \mid 1)\right|_{K_{1} \rightarrow-K_{1}, M_{0} \leftrightarrow M_{1}}\right] \\
= & \tilde{f}_{1}\left(\frac{4\left(5 D^{2}+6 D-8\right) M_{1}^{2} s_{01}^{4}}{D\left(D^{2}-1\right) s_{11}^{3}}+\frac{12 s_{00}\left((2 D-1) M_{1}^{2} s_{00}+2 D(D+1) s_{01}^{2}\right)}{D\left(D^{2}-1\right) s_{11}}\right) \\
& -\tilde{f}_{1} \frac{24\left(\left(D^{2}+2 D-2\right) M_{1}^{2} s_{00} s_{01}^{2}+D^{2}(D+1) s_{01}^{4}\right)}{D\left(D^{2}-1\right) s_{11}^{2}} \\
& +\tilde{f}_{1}^{3}\left(-\frac{\left(D^{2}+6 D+8\right) s_{01}^{4}}{\left(D^{2}-1\right) s_{11}^{4}}+\frac{6(D+2) s_{00} s_{01}^{2}}{\left(D^{2}-1\right) s_{11}^{3}}-\frac{3 s_{00}^{2}}{\left(D^{2}-1\right) s_{11}^{2}}\right) \\
& +\tilde{f}_{1}^{2}\left(\frac{8(D+2) s_{01}^{4}}{(D-1) s_{11}^{3}}-\frac{24 s_{00} s_{01}^{2}}{(D-1) s_{11}^{2}}\right)-\frac{64 M_{1}^{2} s_{01}^{4}}{D s_{11}^{2}}+\frac{32 s_{01}^{2}\left(\frac{3 M_{1}^{2} s_{00}}{D}+s_{01}^{2}\right)}{s_{11}}
\end{aligned}
$$

where $\tilde{f}_{1}=K_{1}^{2}+M_{1}^{2}-M_{0}^{2}$.

The reduction coefficients of bubble: $I_{2}[0,1]$

The expansion of $C^{(0,1)}(4 \mid 1)$ is

$$
C^{(0,1)}(4 \mid 1)=c_{0}^{(0,1)}(4) M_{0}^{4} s_{00}^{2}+c_{2}^{(0,1)}(4) M_{0}^{2} s_{00} s_{01}^{2}+c_{4}^{(0,1)}(4) s_{01}^{4} .
$$

By setting $r=2$ in (4.5), we have

$$
\begin{aligned}
c_{0}^{(0,1)}(4) & =\frac{4}{D+1}\left(4-\frac{\alpha_{1}^{2}}{\beta_{11}}\right) c_{0}^{(0,1)}(2)=\frac{4}{D+1}\left[\left(4-\frac{\alpha_{1}^{2}}{\beta_{11}}\right)\left(\frac{4}{D-1}-\frac{f_{1}^{2}}{(D-1) M_{0}^{2} s_{11}}\right)\right] \\
& =\frac{3 f_{1}^{4}}{\left(D^{2}-1\right) M_{0}^{4} s_{11}^{2}}-\frac{24 f_{1}^{2}}{\left(D^{2}-1\right) M_{0}^{2} s_{11}}+\frac{48}{D^{2}-1} .
\end{aligned}
$$

By setting $i=0,2$ in (4.4), we calculate $c_{2}^{(0,1)}(4)$ and $c_{4}^{(0,1)}(4)$ iteratively,

$$
\begin{aligned}
c_{2}^{(0,1)}(4) & =\frac{1}{2 \beta_{11}}\left(4 \alpha_{1} c_{1}^{(0,1)}(3)-4 c_{0}^{(0,1)}(4)\right) \\
& =\frac{1}{2 \beta_{11}}\left[4 \alpha_{1}\left(\frac{(D+2) f_{1}^{3}}{(D-1) s_{11}^{3}}-\frac{12 f_{1} M_{0}^{2}}{(D-1) s_{11}^{2}}\right)-4\left(\frac{3 f_{1}^{4}}{\left(D^{2}-1\right) M_{0}^{4} s_{11}^{2}}-\frac{24 f_{1}^{2}}{\left(D^{2}-1\right) M_{0}^{2} s_{11}}+\frac{48}{D^{2}-1}\right)\right] \\
& =-\frac{6(D+2) f_{1}^{4}}{\left(D^{2}-1\right) M_{0}^{2} s_{11}^{3}}+\frac{24(D+3) f_{1}^{2}}{\left(D^{2}-1\right) s_{11}^{2}}-\frac{96 M_{0}^{2}}{\left(D^{2}-1\right) s_{11}},
\end{aligned}
$$




$$
\begin{aligned}
c_{4}^{(0,1)}(4) & =\frac{1}{4 \beta_{11}}\left(4 \alpha_{1} c_{3}^{(0,1)}(3)-2 c_{2}^{(0,1)}(4)\right) \\
& =\frac{1}{4 \beta_{11}}\left[4 \alpha_{1}\left(\frac{(D+2) f_{1}^{3}}{(D-1) s_{11}^{3}}-\frac{12 f_{1} M_{0}^{2}}{(D-1) s_{11}^{2}}\right)-2\left(-\frac{6(D+2) f_{1}^{4}}{\left(D^{2}-1\right) M_{0}^{2} s_{11}^{3}}+\frac{24(D+3) f_{1}^{2}}{\left(D^{2}-1\right) s_{11}^{2}}-\frac{96 M_{0}^{2}}{\left(D^{2}-1\right) s_{11}}\right)\right] \\
& =-\frac{24(D+2) f_{1}^{2} M_{0}^{2}}{\left(D^{2}-1\right) s_{11}^{3}}+\frac{\left(D^{2}+6 D+8\right) f_{1}^{4}}{\left(D^{2}-1\right) s_{11}^{4}}+\frac{48 M_{0}^{4}}{\left(D^{2}-1\right) s_{11}^{2}},
\end{aligned}
$$

where we have used the results of the expansion coefficients with lower rank. Then the reduction coefficient is

$$
\begin{aligned}
C^{(0,1)}(4 \mid 1)= & -\frac{24 f_{1}^{2} M_{0}^{2}\left(s_{01}^{2}-s_{00} s_{11}\right)\left((D+2) s_{01}^{2}-s_{00} s_{11}\right)}{\left(D^{2}-1\right) s_{11}^{3}}+\frac{48 M_{0}^{4}\left(s_{01}^{2}-s_{00} s_{11}\right)^{2}}{\left(D^{2}-1\right) s_{11}^{2}} \\
& +\frac{f_{1}^{4}\left(\left(D^{2}+6 D+8\right) s_{01}^{4}-6(D+2) s_{00} s_{11} s_{01}^{2}+3 s_{00}^{2} s_{11}^{2}\right)}{\left(D^{2}-1\right) s_{11}^{4}}
\end{aligned}
$$

\section{B. Reduce tensor box to scalar triangles}

We will consider the reduction coefficients of triangle MIs of tensor integral $I_{4}^{(1)}$ as another example to illustrate the algorithm in Sec. III B. For simplicity, we denote $G\left(i_{1}, i_{2}, \ldots, i_{s} ; j_{1}, j_{2}, \ldots, j_{r}\right)$ as the determinant of the Gram matrix $G$ with entries $G_{a b}=K_{a} \cdot K_{b}=s_{a b}$. Specially, we denote $G\left(i_{1}, i_{2}, \ldots, i_{s}\right) \equiv G\left(i_{1}, i_{2}, \ldots, i_{s} ; i_{1}, i_{2}, \ldots, i_{s}\right)$.

The reduction coefficient of the scalar triangle $I_{3}[0,1,2]$ is

$$
C^{(0,1,2)}(1 \mid 3)=-\frac{G(2,3 ; 1,2) s_{01}-G(1,3 ; 1,2) s_{02}+G(1,2 ; 1,2) s_{03}}{G(1,2,3)} .
$$

The reduction coefficients of $I_{3}[0,1,3]$ and $I_{3}[0,2,3]$ are easy to obtained by simply changing labels $\{1,2,3\} \rightarrow\{1,3,2\}$ and $\{1,2,3\} \rightarrow\{2,1,3\}$ respectively,

$$
\begin{aligned}
C^{(0,1,3)}(1 \mid 3) & =\left.C^{(0,1,2)}(1 \mid 3)\right|_{K_{2} \leftrightarrow K_{3}, M_{2} \leftrightarrow M_{3}} \\
& =-\frac{G(3,2 ; 1,3) s_{01}-G(1,2 ; 1,3) s_{03}+G(1,3 ; 1,3) s_{02}}{G(1,2,3)}, \\
C^{(0,2,3)}(1 \mid 3) & =\left.C^{(0,1,2)}(1 \mid 3)\right|_{K_{1} \leftrightarrow K_{2}, M_{1} \leftrightarrow M_{2}} \\
& =-\frac{G(2,1 ; 3,2) s_{03}-G(3,1 ; 3,2) s_{02}+G(3,2 ; 3,2) s_{01}}{G(1,2,3)} .
\end{aligned}
$$

Now we consider the reduction coefficient of the triangle without $P_{0}$, i.e., $I_{3}[1,2,3]$. In (3.16), choosing $j_{0}=3, j_{1}=1$, $j_{2}=2$, we have

$$
\begin{aligned}
C^{(1,2,3)}(1 \mid 3)= & C^{(3,1,2)}(1 \mid 3) \\
= & \left.\left(2 R \cdot K_{3}\right) C^{(0,1,2)}(0 \mid 3)\right|_{K_{1} \rightarrow K_{1}-K_{3}, K_{2} \rightarrow K_{2}-K_{3}, K_{3} \rightarrow-K_{3}, M_{0} \leftrightarrow M_{3}}+\left.C^{(0,1,2)}(1 \mid 3)\right|_{K_{1} \rightarrow K_{1}-K_{3}, K_{2} \rightarrow K_{2}-K_{3}, K_{3} \rightarrow-K_{3}, M_{0} \leftrightarrow M_{3}} \\
= & \frac{G\left(K_{2}-K_{3}, K_{3} ; K_{1}-K_{3}, K_{2}-K_{3}\right)\left(s_{01}-s_{03}\right)}{G\left(K_{1}-K_{3}, K_{2}-K_{3}, K_{3} ; K_{1}-K_{3}, K_{2}-K_{3}, K_{3}\right)}+\frac{-G\left(K_{1}-K_{3}, K_{3} ; K_{1}-K_{3}, K_{2}-K_{3}\right)\left(s_{02}-s_{03}\right)}{G\left(K_{1}-K_{3}, K_{2}-K_{3}, K_{3} ; K_{1}-K_{3}, K_{2}-K_{3}, K_{3}\right)} \\
& +\frac{G\left(K_{1}-K_{3}, K_{2}-K_{3} ; K_{1}-K_{3}, K_{2}-K_{3}\right) s_{03}}{G\left(K_{1}-K_{3}, K_{2}-K_{3}, K_{3} ; K_{1}-K_{3}, K_{2}-K_{3}, K_{3}\right)}
\end{aligned}
$$

where $C^{(0,1,2)}(0 \mid 3)=0$ is the reduction coefficient of a triangle MI from a box MI. 


\section{DISCUSSION}

In this paper we show how to use the differential operators to get the analytical expressions for the reduction coefficients of all master basis. Using these operators, one can establish the recurrence relations about reduction coefficients in differential equation form. Another crucial step in this method is that we use the information of tensor structure to avoid solving the intricate differential equations directly.

As we have reviewed, in [31-33], the analytical expressions for reduction coefficients can be solved by the unitarity method. However, there are some differences between these two approaches.

(a) The first difference is that the expression given by the unitarity cut method is written using the spinor formalism, while the results in this paper use the traditional Lorentz-invariant contractions.

(b) The second difference is that in the unitarity cut method, we have assumed the external momenta to be purely $4 D$ and the only loop momentum is in general $(4-2 \epsilon)$ dimensions. For our new method, there is no such a constraint and the external momenta can be in $4 D$ or in $(4-2 \epsilon)$ dimensions.

(c) The third difference is that results in this paper are defined in an iterated way, while expressions given by the unitarity cut method are just one equation (although the differentiation has the spirit of iteration).

(d) The fourth difference is that expressions of the unitarity cut method use input of arbitrary forms, while the one in this paper uses the standard input given in (2.2). The difference has a potentially huge impact on the computation efficiency. The reason is that with the development of the on-shell program, it is well known that tree-level amplitudes will be significantly simplified if we use spinor variables with spurious poles, such as these given by the recursion relation $[37,38]$. Thus, it will be desirable to incorporate these advantages of the unitarity cut method to our current new strategy.
Finally, let us emphasize that the purpose of this paper is to establish an independent and complete reduction framework for one-loop integrals using the auxiliary vector $R$. In our previous work [36], we discussed the reduction coefficients of tadpoles. In this paper we have completed the coefficients of other basis. However, for these two works, we have assumed the power of each propagator is just one. To be a complete reduction framework we need to find the reduction of integrals with arbitrary tensor structures, and propagators having arbitrary powers. We will show in an upcoming paper how to achieve this. After completing the framework of reduction with the auxiliary vector $R$, we can discuss various limit cases, like the massless limit or the vanishing of Gram determinant, which will be presented in another paper. Another direction is to apply our new framework to higher loops. But unlike the one-loop case, relations established by differentiation over $R$ are usually not enough. Besides, the master integrals are more complicated in higher-loop integrals. How to solve these difficulties will be another future project.

\section{ACKNOWLEDGMENTS}

We are very grateful for Bo Feng's collaboration in the previous related paper and inspiring discussion. This work is supported by Qiu-Shi Funding and Chinese NSF funding under Grants No. 11935013, No. 11947301, and No. 12047502 (Peng Huanwu Center).

\section{APPENDIX: MORE EXAMPLES}

In this Appendix we provide more examples to illustrate our method. ${ }^{4}$ There are three points we need to emphasize ahead.

(a) For the tensor integral $I_{n+1}^{(m)}$, we only list the reduction coefficient $C^{i_{r}}$ for $m \geq n-\left|i_{r}\right|$, because there are not enough $\ell \cdot R$ in the numerator to cancel $n-\left|i_{r}\right|$ propagators for $m<n-\left|i_{r}\right|$.

(b) We merely list the results of $C^{(0,1, \ldots, r)}(m \mid n)$, $C^{(1,2, \ldots, r+1)}(m \mid n), 0 \leq r \leq 4$ due to the permutation symmetry,

$$
\begin{aligned}
\text { Tadpoles: } C^{(i)}(m \mid n) & =\left.C^{(1)}(m \mid n)\right|_{1 \leftrightarrow i} \\
\text { Bubbles: } C^{(0, i)}(m \mid n) & =\left.C^{(0,1)}(m \mid n)\right|_{1 \leftrightarrow i} \\
C^{(i, j)}(m \mid n) & =\left.C^{(1,2)}(m \mid n)\right|_{1 \leftrightarrow i, 2 \leftrightarrow j} \\
\text { Triangles: } C^{(0, i, j)}(m \mid n)= & \left.C^{(0,1,2)}(m \mid n)\right|_{1 \leftrightarrow i, 2 \leftrightarrow j} \\
C^{(i, j, k)}(m \mid n)= & \left.C^{(1,2,3)}(m \mid n)\right|_{1 \leftrightarrow i, 2 \leftrightarrow j, 3 \leftrightarrow k} \\
\text { Boxes: } C^{(0, i, j, k)}(m \mid n)= & \left.C^{(0,1,2,3)}(m \mid n)\right|_{1 \leftrightarrow i, 2 \leftrightarrow j, 3 \leftrightarrow k} \\
C^{(i, j, k, l)}(m \mid n)= & \left.C^{(1,2,3,4)}(m \mid n)\right|_{1 \leftrightarrow i, 2 \leftrightarrow j, 3 \leftrightarrow k, 4 \leftrightarrow l} \\
& \ldots
\end{aligned}
$$

\footnotetext{
${ }^{4}$ All results have been checked with Fire6 [39-43].
} 
where $0<i<j<k<l$.

(c) There is a permutation symmetry about the expansion coefficient $c_{\left.a_{1}, \ldots, j_{n}\right)}^{\left(j_{0}, \ldots, j_{r}\right)}(m)$ If the master integral $I_{r+1}\left[j_{0}, j_{1}, \ldots, j_{r}\right]$ is invariant under a label permutation $\sigma:\{1,2, \ldots, n\} \rightarrow\{\sigma(1), \sigma(2), \ldots, \sigma(n)\}, \quad$ we have $c_{i_{1}, \ldots, i_{n}}^{\left(j_{0}, \ldots, j_{r}\right)}(m)=\sigma c_{i_{\sigma^{-1}(1)}, \ldots, i_{\sigma^{-1}(n)}}^{\left(j_{0}, \ldots, j_{r}\right)}(m)$. For example, $I_{4}[0,1,2,3]$ is invariant under the label permutation $\sigma:\{1,2,3,4\} \rightarrow\{3,1,2,4\}$, then we have

$$
c_{1,2,4,5}^{(0,1,2,3)}(14)=\left.c_{2,4,1,5}^{(0,1,2,3)}(14)\right|_{\{1,2,3,4\} \rightarrow\{3,1,2,4\}} .
$$

\section{All reduction coefficients of tensor triangle with rank $m=1,2$}

The MI of a tensor triangle $I_{3}^{(m)}$ are

Tadpoles: $I_{1}[0], I_{1}[1], I_{1}[2]$,

Bubbles: $I_{2}[0,1], I_{2}[0,2], I_{2}[1,2]$,

Triangles: $I_{3}[0,1,2]$.

Then the reduction of the tensor triangle is

$$
\begin{aligned}
I_{3}^{(m)}= & C^{(0)}(m \mid 2) I_{1}[0]+C^{(1)}(m \mid 2) I_{1}[1]+C^{(2)}(m \mid 2) I_{1}[2]+C^{(0,1)}(m \mid 2) I_{2}[0,1] \\
& +C^{(0,2)} I_{2}[0,2]+C^{(1,2)} I_{2}[1,2]+C^{(0,1,2)} I_{3}[0,1,2] .
\end{aligned}
$$

(a) $m=1$

Reduction coefficients of tadpoles

All reduction coefficients vanish.

Reduction coefficient of bubbles

$$
C^{(0,1)}(1 \mid 2)=\frac{s_{01} s_{12}-s_{02} s_{11}}{G(1,2)} .
$$

Choosing $j_{0}=1, j_{1}=2$ in (3.16), we have

$$
\begin{aligned}
C^{(1,2)}(1 \mid 2) & =\left.C^{(0,2)}(1 \mid 2)\right|_{M_{0} \leftrightarrow M_{1}, K_{1} \rightarrow-K_{1}, K_{2} \rightarrow K_{2}-K_{1}} \\
& =\left.\frac{s_{02} s_{12}-s_{01} s_{22}}{G(1,2)}\right|_{M_{0} \leftrightarrow M_{1}, K_{1} \rightarrow-K_{1}, K_{2} \rightarrow K_{2}-K_{1}} \\
& =\frac{s_{02}\left(s_{11}-s_{12}\right)}{G(1,2)}+(1 \leftrightarrow 2) .
\end{aligned}
$$

Reduction coefficient of triangle

$$
C^{(0,1,2)}(1 \mid 2)=\frac{s_{01}\left(f_{1} s_{22}-f_{2} s_{12}\right)}{G(1,2)}+(1 \leftrightarrow 2) .
$$

(b) $m=2$

Reduction coefficients of tadpoles

$$
C^{(0)}(2 \mid 2)=\frac{s_{11} s_{22} s_{01} s_{02}-s_{12} s_{22} s_{01}^{2}}{s_{11} s_{22} G(1,2)}+(1 \leftrightarrow 2) .
$$

Choosing $j_{0}=1$ in (3.16), we have

$$
\begin{aligned}
C^{(1)}(2 \mid 2) & =\left.C^{(0)}(2 \mid 2)\right|_{M_{0} \leftrightarrow M_{1}, K_{1} \rightarrow-K_{1}, K_{2} \rightarrow K_{2}-K_{1}} \\
& =-\left.\frac{s_{12} s_{22} s_{01}^{2}-2 s_{02} s_{11} s_{22} s_{01}+s_{02}^{2} s_{11} s_{12}}{s_{11} s_{22}\left(s_{11} s_{22}-s_{12}^{2}\right)}\right|_{M_{0} \leftrightarrow M_{1}, K_{1} \rightarrow-K_{1}, K_{2} \rightarrow K_{2}-K_{1}} \\
& =\frac{\left(-2 s_{12}^{2}+s_{22} s_{12}+s_{11} s_{22}\right) s_{01}^{2}+2 s_{02} s_{11}\left(s_{12}-s_{22}\right) s_{01}+s_{02}^{2} s_{11}\left(s_{12}-s_{11}\right)}{s_{11}\left(s_{11}-2 s_{12}+s_{22}\right) G(1,2)} .
\end{aligned}
$$


Reduction coefficients of bubbles

$$
C^{(0,1)}(2 \mid 2)=\frac{s_{01}^{2} c_{2,0}^{(0,1)}(2)}{M_{0}^{2}}+\frac{s_{02} s_{01} c_{1,1}^{(0,1)}(2)}{M_{0}^{2}}+\frac{s_{02}^{2} c_{0,2}^{(0,1)}(2)}{M_{0}^{2}}+s_{00} c_{0,0}^{(0,1)}(2),
$$

where

$$
\begin{gathered}
c_{0,0}^{(0,1)}(2)=\frac{f_{2} s_{11}-f_{1} s_{12}}{(D-2) G(1,2)}, \\
c_{1,1}^{(0,1)}(2)=\frac{2(D-1) f_{2} M_{0}^{2} s_{11} s_{22}}{(D-2)\left(s_{12}^{2}-s_{11} s_{22}\right)^{2}}-\frac{2 f_{1} M_{0}^{2}\left((D-2) f_{2} M_{0}^{2} s_{11} s_{22}+s_{12}^{2}\right)}{(D-2)\left(s_{12}^{2}-s_{11} s_{22}\right)^{2}}, \\
c_{2,0}^{(0,1)}(2)=-\frac{M_{0}^{2}\left(f_{2} s_{11}\left((D-2) s_{12}^{2}+s_{11} s_{22}\right)+f_{1} s_{12}\left((D-2) s_{12}^{2}+(3-2 D) s_{11} s_{22}\right)\right)}{(D-2) s_{11} G(1,2)^{2}}, \\
c_{0,2}^{(0,1)}(2)=-\frac{(D-1) M_{0}^{2} s_{11}\left(f_{2} s_{11}-f_{1} s_{12}\right)}{(D-2)^{2} G(1,2)^{2}} \\
C^{(1,2)}(2 \mid 2)=2\left(2 R \cdot K_{2}\right)\left[\left.C^{(0,1)}(1,2)\right|_{\sigma}\right]+\left.C^{(0,1)}(2,2)\right|_{\sigma} \\
=4 s_{02}\left[\frac{s_{01}\left(f_{1} s_{22}-f_{2} s_{12}\right)}{G(1,2)}+(1 \leftrightarrow 2)\right]+\left.C^{(0,1)}(2,2)\right|_{\sigma}
\end{gathered}
$$

where

$$
\sigma=M_{0} \leftrightarrow M_{2}, K_{2} \rightarrow-K_{2}, K_{1} \rightarrow K_{1}-K_{2} .
$$

Reduction coefficients of triangle

$$
C^{(0,1,2)}(2 \mid 2)=c_{0,0}^{(0,1,2)} M_{0}^{2} s_{00}+c_{2,0}^{(0,1,2)} s_{01}^{2}+c_{0,2}^{(0,1,2)} s_{02}^{2}+c_{1,1}^{(0,1,2)} s_{01} s_{02} .
$$

where

$$
\begin{aligned}
c_{0,0}^{(0,1,2)}(2)= & \frac{f_{1}^{2} s_{22}-2 f_{2} f_{1} s_{12}+f_{2}^{2} s_{11}+4 M_{0}^{2}\left(s_{12}^{2}-s_{11} s_{22}\right)}{(D-2) M_{0}^{2}\left(s_{12}^{2}-s_{11} s_{22}\right)}, \\
c_{2,0}^{(0,1,2)}(2)= & \frac{f_{2}^{2}\left((D-2) s_{12}^{2}+s_{11} s_{22}\right)}{(D-2)\left(s_{12}^{2}-s_{11} s_{22}\right)^{2}}-\frac{2(D-1) f_{1} f_{2} s_{12} s_{22}}{(D-2)\left(s_{12}^{2}-s_{11} s_{22}\right)^{2}} \\
& +\frac{(D-1) f_{1}^{2} s_{22}^{2}}{(D-2)\left(s_{12}^{2}-s_{11} s_{22}\right)^{2}}+\frac{4 M_{0}^{2} s_{22}}{(D-2)\left(s_{12}^{2}-s_{11} s_{22}\right)}, \\
c_{0,2}^{(0,1,2)}(2)= & \frac{f_{1}^{2}\left((D-2) s_{12}^{2}+s_{11} s_{22}\right)}{(D-2)\left(s_{12}^{2}-s_{11} s_{22}\right)^{2}}-\frac{2(D-1) f_{2} f_{1} s_{11} s_{12}}{(D-2)\left(s_{12}^{2}-s_{11} s_{22}\right)^{2}} \\
& +\frac{(D-1) f_{2}^{2} s_{11}^{2}}{(D-2)\left(s_{12}^{2}-s_{11} s_{22}\right)^{2}}+\frac{4 M_{0}^{2} s_{11}}{(D-2)\left(s_{12}^{2}-s_{11} s_{22}\right)},
\end{aligned}
$$




$$
\begin{aligned}
c_{1,1}^{(0,1,2)}(2)= & -\frac{2(D-1) f_{1}^{2} s_{12} s_{22}}{(D-2)\left(s_{12}^{2}-s_{11} s_{22}\right)^{2}}+\frac{2 f_{2} f_{1}\left(D s_{12}^{2}+(D-2) s_{11} s_{22}\right)}{(D-2)\left(s_{12}^{2}-s_{11} s_{22}\right)^{2}} \\
& -\frac{2(D-1) f_{2}^{2} s_{11} s_{12}}{(D-2)\left(s_{12}^{2}-s_{11} s_{22}\right)^{2}}-\frac{8 M_{0}^{2} s_{12}}{(D-2)\left(s_{12}^{2}-s_{11} s_{22}\right)} .
\end{aligned}
$$

\section{All reduction coefficients of tensor box with rank $m=1,2$}

The MIs of a tensor box $I_{4}^{(m)}$ are

Tadpoles: $I_{1}[0], I_{1}[1], I_{1}[2], I_{1}[3]$,

Bubbles: $I_{2}[0,1], I_{2}[0,2], I_{2}[0,3], I_{2}[1,2], I_{2}[1,3], I_{2}[2,3]$,

Triangles: $I_{3}[0,1,2], I_{3}[0,1,3], I_{3}[0,2,3], I_{3}[1,2,3]$,

Box: $I_{4}[0,1,2,3]$.

Then the reduction of the tensor box is

$$
\begin{aligned}
I_{4}^{(m)}= & \sum_{i=0}^{3} C^{(i)}(m \mid 3) I_{1}[i]+\sum_{0 \leq i_{1}<i_{2} \leq 3} C^{\left(i_{1}, i_{2}\right)}(m \mid 3) I_{2}\left[i_{1}, i_{2}\right] \\
& +\sum_{0 \leq i_{1}<i_{2}<i_{3} \leq 3} C^{\left(i_{1}, i_{2}, i_{3}\right)}(m \mid 3) I_{3}\left[i_{1}, i_{2}, i_{3}\right]+C^{(0,1,2,3)} I_{4}[0,1,2,3] .
\end{aligned}
$$

(a) $m=1$

Reduction coefficients of tadpoles, bubbles

All reduction coefficients vanish.

Reduction coefficients of triangles

$$
C^{(0,1,2)}(1 \mid 3)=-\frac{G(2,3 ; 1,2) s_{01}-G(1,3 ; 1,2) s_{02}+G(1,2 ; 1,2) s_{03}}{G(1,2,3)} .
$$

In (3.16), choosing $j_{0}=3, j_{1}=1, j_{2}=2$, we have

$$
\begin{aligned}
C^{(1,2,3)}(1 \mid 3)= & C^{(3,1,2)}(1 \mid 3) \\
= & \left.\left(2 R \cdot K_{3}\right) C^{(0,1,2)}(0 \mid 3)\right|_{K_{1} \rightarrow K_{1}-K_{3}, K_{2} \rightarrow K_{2}-K_{3}, K_{3} \rightarrow-K_{3}, M_{0} \leftrightarrow M_{3}}+\left.C^{(0,1,2)}(1 \mid 3)\right|_{K_{1} \rightarrow K_{1}-K_{3}, K_{2} \rightarrow K_{2}-K_{3}, K_{3} \rightarrow-K_{3}, M_{0} \leftrightarrow M_{3}} \\
= & \frac{G\left(K_{2}-K_{3}, K_{3} ; K_{1}-K_{3}, K_{2}-K_{3}\right)\left(s_{01}-s_{03}\right)}{G\left(K_{1}-K_{3}, K_{2}-K_{3}, K_{3} ; K_{1}-K_{3}, K_{2}-K_{3}, K_{3}\right)}+\frac{-G\left(K_{1}-K_{3}, K_{3} ; K_{1}-K_{3}, K_{2}-K_{3}\right)\left(s_{02}-s_{03}\right)}{G\left(K_{1}-K_{3}, K_{2}-K_{3}, K_{3} ; K_{1}-K_{3}, K_{2}-K_{3}, K_{3}\right)} \\
& +\frac{G\left(K_{1}-K_{3}, K_{2}-K_{3} ; K_{1}-K_{3}, K_{2}-K_{3}\right) s_{03}}{G\left(K_{1}-K_{3}, K_{2}-K_{3}, K_{3} ; K_{1}-K_{3}, K_{2}-K_{3}, K_{3}\right)}
\end{aligned}
$$

Reduction coefficients of box

$$
\begin{aligned}
C^{(0,1,2,3)}(1 \mid 3)= & \frac{f_{3}\left(s_{01} G(2,3 ; 1,2)-s_{02} G(1,3 ; 1,2)+s_{03} G(1,2 ; 1,2)\right)}{G(1,2,3)} \\
& -\frac{f_{2}\left(s_{01} G(2,3 ; 1,3)-s_{02} G(1,3 ; 1,3)+s_{03} G(1,3 ; 1,2)\right)}{G(1,2,3)} \\
& +\frac{f_{1}\left(s_{01} G(2,3 ; 2,3)-s_{02} G(2,3 ; 1,3)+s_{03} G(2,3 ; 1,2)\right)}{G(1,2,3)}
\end{aligned}
$$


(b) $m=2$

Reduction coefficients of tadpoles

All reduction coefficients vanish.

Reduction coefficients of bubbles

$$
\begin{aligned}
C^{(0,1)}(2 \mid 3)= & \left(M_{0}^{2}\right)^{-2}\left[c_{0,0,0}^{(0,1)}(2) M_{0}^{2} s_{00}+c_{2,0,0}^{(0,1)}(2) s_{01}^{2}+c_{0,2,0}^{(0,1)}(2) s_{02}^{2}+c_{0,0,2}^{(0,1)}(2) s_{03}^{2}\right. \\
& \left.+c_{1,1,0}^{(0,1)}(2) s_{01} s_{02}+c_{0,1,1}^{(0,1)}(2) s_{02} s_{03}+c_{1,0,1}^{(0,1)}(2) s_{01} s_{03}\right] .
\end{aligned}
$$

where

$$
\begin{gathered}
c_{0,0,0}^{(0,1)}(2)=0, \\
c_{2,0,0}^{(0,1)}(2)=\frac{M_{0}^{4} s_{13} G(2,3 ; 1,3)}{G(1,3 ; 1,3) G(1,2,3)}-\frac{M_{0}^{4} s_{12} G(2,3 ; 1,2)}{G(1,2 ; 1,2) G(1,2,3)}, \\
c_{0,2,0}^{(0,1)}(2)=-\frac{M_{0}^{4} s_{11} G(1,3 ; 1,2)}{G(1,2 ; 1,2) G(1,2,3)}, \\
c_{0,0,2}^{(0,1)}(2)=\left.c_{0,2,0}^{(0,1)}(2)\right|_{2 \leftrightarrow 3}, \\
c_{0,1,1}^{(0,1)}(2)=\frac{2 M_{0}^{4} s_{11}}{G(1,2,3)}, \\
c_{1,1,0}^{(0,1)}(2)=\frac{2 M_{0}^{4} s_{11} G(2,3 ; 1,2)}{G(1,2 ; 1,2) G(1,2,3)}, \\
c_{1,0,1}^{(0,1)}(2)=\left.c_{1,1,0}^{(0,1)}(2)\right|_{2 \leftrightarrow 3} .
\end{gathered}
$$

Choosing $j_{0}=2, j_{1}=1$ in (3.16) we have

$$
\begin{aligned}
C^{(1,2)}(2 \mid 3) & =\left.\left[2\left(-2 R \cdot K_{2}\right) C^{(0,1)}(1 \mid 3)+C^{(0,1)}(2 \mid 3)\right]\right|_{K_{1} \rightarrow K_{1}-K_{2}, K_{2} \rightarrow-K_{2}, K_{3} \rightarrow K_{3}-K_{2} M_{0} \leftrightarrow M_{2}} \\
& =\left.C^{(0,1)}(2 \mid 3)\right|_{K_{1} \rightarrow K_{1}-K_{2}, K_{2} \rightarrow-K_{2}, K_{3} \rightarrow K_{3}-K_{2} M_{0} \leftrightarrow M_{2} .}
\end{aligned}
$$

Reduction coefficients of triangles

$$
\begin{aligned}
C^{(0,1,2)}(2 \mid 3)= & M_{0}^{-2}\left[c_{0,0,0}^{(0,1,2)}(2) M_{0}^{2} s_{00}+c_{2,0,0}^{(0,1,2)}(2) s_{01}^{2}+c_{0,2,0}^{(0,1,2)}(2) s_{02}^{2}+c_{0,0,2}^{(0,1,2)}(2) s_{03}^{2}\right. \\
& \left.+c_{1,1,0}^{(0,1,2)}(2) s_{01} s_{02}+c_{0,1,1}^{(0,1,2)}(2) s_{02} s_{03}+c_{1,0,1}^{(0,1,2)}(2) s_{01} s_{03}\right]
\end{aligned}
$$

where

$$
c_{0,0,0}^{(0,1,2)}(2)=\frac{f_{1} G(2,3 ; 1,2)}{(D-3) G(1,2,3)}-\frac{f_{2} G(1,3 ; 1,2)}{(D-3) G(1,2,3)}+\frac{f_{3} G(1,2 ; 1,2)}{(D-3) G(1,2,3)},
$$




$$
\begin{aligned}
& c_{2,0,0}^{(0,1,2)}(2)=\frac{f_{2} M_{0}^{2} G(1,3 ; 1,2) G(2,3 ; 2,3)}{(D-3) G(1,2,3)^{2}}-\frac{f_{3} M_{0}^{2} G(1,2 ; 1,2) G(2,3 ; 2,3)}{(D-3) G(1,2,3)^{2}} \\
& +G(2,3 ; 1,2)\left(\frac{f_{2} M_{0}^{2} G(2,3 ; 1,3)}{G(1,2,3)^{2}}-\frac{(D-2) f_{1} M_{0}^{2} G(2,3 ; 2,3)}{(D-3) G(1,2,3)^{2}}\right) \\
& +\frac{M_{0}^{2}\left(f_{2} s_{12}-f_{1} s_{22}\right) G(2,3 ; 1,2)}{G(1,2 ; 1,2) G(1,2,3)}-\frac{f_{3} M_{0}^{2} G(2,3 ; 1,2)^{2}}{G(1,2,3)^{2}}, \\
& c_{0,2,0}^{(0,1,2)}(2)=\left.c_{2,0,0}^{(0,1,2)}(2)\right|_{1 \leftrightarrow 2}, \\
& c_{0,0,2}^{(0,1,2)}(2)=G(1,2 ; 1,2)\left[\frac{(D-2) f_{2} M_{0}^{2} G(1,3 ; 1,2)}{(D-3) G(1,2,3 ; 1,2,3)^{2}}-\frac{(D-2) f_{1} M_{0}^{2} G(2,3 ; 1,2)}{(D-3) G(1,2,3 ; 1,2,3)^{2}}\right] \\
& -\frac{(D-2) f_{3} M_{0}^{2} G(1,2 ; 1,2)^{2}}{(D-3) G(1,2,3 ; 1,2,3)^{2}} \\
& c_{1,1,0}^{(0,1,2)}(2)=-\frac{2(D-2) f_{2} M_{0}^{2} G(1,3 ; 1,2) G(2,3 ; 1,3)}{(D-3) G(1,2,3)^{2}}+\frac{2 f_{3} M_{0}^{2} G(1,2 ; 1,2) G(2,3 ; 1,3)}{(D-3) G(1,2,3)^{2}} \\
& +G(2,3 ; 1,2)\left(\frac{2 f_{1} M_{0}^{2} G(2,3 ; 1,3)}{(D-3) G(1,2,3)^{2}}+\frac{2 f_{3} M_{0}^{2} G(1,3 ; 1,2)}{G(1,2,3)^{2}}\right) \\
& +\frac{2 M_{0}^{2}\left(f_{1} s_{12}-f_{2} s_{11}\right) G(2,3 ; 1,2)}{G(1,2 ; 1,2) G(1,2,3)}+\frac{2 f_{1} M_{0}^{2} G(1,3 ; 1,2) G(2,3 ; 2,3)}{G(1,2,3)^{2}}, \\
& c_{0,1,1}^{(0,1,2)}(2)=\frac{2 f_{1} M_{0}^{2} G(1,3 ; 1,2) G(2,3 ; 1,2)}{(D-3) G(1,2,3)^{2}}-\frac{2 f_{2} M_{0}^{2} G(1,3 ; 1,2)^{2}}{(D-3) G(1,2,3)^{2}} \\
& +G(1,2 ; 1,2)\left(\frac{2(D-2) f_{3} M_{0}^{2} G(1,3 ; 1,2)}{(D-3) G(1,2,3)^{2}}+\frac{2 f_{1} M_{0}^{2} G(2,3 ; 1,3)}{G(1,2,3)^{2}}\right) \\
& -\frac{2 f_{2} M_{0}^{2} G(1,2 ; 1,2) G(1,3 ; 1,3)}{G(1,2,3)^{2}} \\
& c_{1,0,1}^{(0,1,2)}(2)=\left.c_{0,1,1}^{(0,1,2)}(2)\right|_{1 \leftrightarrow 2} .
\end{aligned}
$$

Choosing $j_{0}=3, j_{1}=1, j_{2}=2$ in (3.16), we have

$$
\begin{aligned}
C^{(1,2,3)}(1 \mid 3)= & {\left.\left[2\left(-2 R \cdot K_{3}\right) C^{(0,1,2)}(1 \mid 3)+C^{(0,1,2)}(2 \mid 3)\right]\right|_{K_{1} \rightarrow K_{1}-K_{3}, K_{2} \rightarrow K_{2}-K_{3}, K_{3} \rightarrow-K_{3}, M_{0} \leftrightarrow M_{3}} } \\
= & 4 s_{03}\left\{\frac{G\left(K_{2}-K_{3}, K_{3} ; K_{1}-K_{3}, K_{2}-K_{3}\right)\left(s_{01}-s_{03}\right)}{G\left(K_{1}-K_{3}, K_{2}-K_{3}, K_{3} ; K_{1}-K_{3}, K_{2}-K_{3}, K_{3}\right)}\right. \\
& +\frac{-G\left(K_{1}-K_{3}, K_{3} ; K_{1}-K_{3}, K_{2}-K_{3}\right)\left(s_{02}-s_{03}\right)}{G\left(K_{1}-K_{3}, K_{2}-K_{3}, K_{3} ; K_{1}-K_{3}, K_{2}-K_{3}, K_{3}\right)} \\
& \left.+\frac{G\left(K_{1}-K_{3}, K_{2}-K_{3} ; K_{1}-K_{3}, K_{2}-K_{3}\right) s_{03}}{G\left(K_{1}-K_{3}, K_{2}-K_{3}, K_{3} ; K_{1}-K_{3}, K_{2}-K_{3}, K_{3}\right)}\right\} \\
& +\left.C^{(0,1,2)}(2,3)\right|_{K_{1} \rightarrow K_{1}-K_{3}, K_{2} \rightarrow K_{2}-K_{3}, K_{3} \rightarrow-K_{3}, M_{0} \leftrightarrow M_{3}} .
\end{aligned}
$$


Reduction coefficient of box

$$
\begin{aligned}
C^{(0,1,2,3)}(2 \mid 3)= & c_{0,0,0}^{(0,1,2,3)}(2) M_{0}^{2} s_{00}+c_{2,0,0}^{(0,1,2,3)}(2) s_{01}^{2}+c_{0,2,0}^{(0,1,2,3)}(2) s_{02}^{2}+c_{0,0,2}^{(0,1,2,3)}(2) s_{03}^{2} \\
& +c_{1,1,0}^{(0,1,2,3)}(2) s_{01} s_{02}+c_{0,1,1}^{(0,1,2,3)}(2) s_{02} s_{03}+c_{1,0,1}^{(0,1,2,3)}(2) s_{01} s_{03},
\end{aligned}
$$

where

$$
\begin{aligned}
c_{0,0,0}^{(0,1,2,3)}(2)= & -\frac{f_{1}^{2} G(2,3 ; 2,3)}{(D-3) M_{0}^{2} G(1,2,3)}+\frac{2 f_{2} f_{1} G(2,3 ; 1,3)}{(D-3) M_{0}^{2} G(1,2,3)}-\frac{2 f_{3} f_{1} G(2,3 ; 1,2)}{(D-3) M_{0}^{2} G(1,2,3)}-\frac{f_{3}^{2} G(1,2 ; 1,2)}{(D-3) M_{0}^{2} G(1,2,3)} \\
& +\frac{f_{2}\left(2 f_{3} G(1,3 ; 1,2)-f_{2} G(1,3 ; 1,3)\right)}{(D-3) M_{0}^{2} G(1,2,3)}+\frac{4}{D-3},
\end{aligned}
$$

$$
\begin{aligned}
& c_{2,0,0}^{(0,1,2,3)}(2)=\frac{f_{3}^{2} G(1,2 ; 1,2) G(2,3 ; 2,3)}{(D-3) G(1,2,3)^{2}}+\frac{2(D-2) f_{1} f_{3} G(2,3 ; 1,2) G(2,3 ; 2,3)}{(D-3) G(1,2,3)^{2}} \\
& -\frac{2(D-2) f_{1} f_{2} G(2,3 ; 1,3) G(2,3 ; 2,3)}{(D-3) G(1,2,3)^{2}}+\frac{f_{2}^{2} G(2,3 ; 1,3)^{2}}{G(1,2,3)^{2}} \\
& +\frac{f_{2}\left(f_{2} G(1,3 ; 1,3) G(2,3 ; 2,3)-2 f_{3} G(1,3 ; 1,2) G(2,3 ; 2,3)\right)}{(D-3) G(1,2,3)^{2}} \\
& -\frac{4 M_{0}^{2} G(2,3 ; 2,3)}{(D-3) G(1,2,3)}+\frac{(D-2) f_{1}^{2} G(2,3 ; 2,3)^{2}}{(D-3) G(1,2,3)^{2}} \\
& +\frac{f_{3}^{2} G(2,3 ; 1,2)^{2}}{G(1,2,3)^{2}}-\frac{2 f_{2} f_{3} G(2,3 ; 1,2) G(2,3 ; 1,3)}{G(1,2,3)^{2}} \\
& c_{1,1,0}^{(0,1,2,3)}(2)=\frac{8 M_{0}^{2} G(2,3 ; 1,3)}{(D-3) G(1,2,3)} \\
& -\frac{2 f_{2}\left((D-2) f_{2} G(1,3 ; 1,3) G(2,3 ; 1,3)-(D-1) f_{3} G(1,3 ; 1,2) G(2,3 ; 1,3)\right)}{(D-3) G(1,2,3)^{2}} \\
& +\frac{2(D-1) f_{1} f_{2} G(2,3 ; 1,3)^{2}}{(D-3) G(1,2,3)^{2}}-\frac{2 f_{3}^{2} G(1,2 ; 1,2) G(2,3 ; 1,3)}{(D-3) G(1,2,3)^{2}} \\
& +\frac{2 f_{1} G(2,3 ; 2,3)\left(f_{2} G(1,3 ; 1,3)-f_{3} G(1,3 ; 1,2)\right)}{G(1,2,3)^{2}} \\
& -\frac{2(D-2) f_{1}^{2} G(2,3 ; 1,3) G(2,3 ; 2,3)}{(D-3) G(1,2,3)^{2}}-\frac{2(D-1) f_{1} f_{3} G(2,3 ; 1,3) G(2,3 ; 1,2)}{(D-3) G(1,2,3)^{2}} \\
& +\frac{2 f_{3} G(2,3 ; 1,2)\left(f_{2} G(1,3 ; 1,3)-f_{3} G(1,3 ; 1,2)\right)}{G(1,2,3)^{2}}
\end{aligned}
$$

Other expansion coefficients are obtained using permutation symmetry, 


$$
\begin{aligned}
& c_{0,2,0}^{(0,1,2,3)}(2)=\left.c_{2,0,0}^{(0,1,2,3)}(2)\right|_{1 \leftrightarrow 2}, \\
& c_{0,0,2}^{(0,1,2,3)}(2)=\left.c_{2,0,0}^{(0,1,2,3)}(2)\right|_{1 \leftrightarrow 3}, \\
& c_{1,0,1}^{(0,1,2,3)}(2)=\left.c_{1,1,0}^{(0,1,2,3)}(2)\right|_{2 \leftrightarrow 3}, \\
& c_{0,1,1}^{(0,1,2,3)}(2)=\left.c_{1,1,0}^{(0,1,2,3)}(2)\right|_{1 \leftrightarrow 3} .
\end{aligned}
$$

\section{All reduction coefficients of tensor pentagon with rank $m=1,2$}

Consider the reduction of a tensor pentagon $I_{5}^{(m)}$; the MIs are

Tadpoles: $I_{1}[0], I_{1}[1], I_{1}[2], I_{1}[3], I_{1}[4]$,

Bubbles: $I_{2}[0,1], I_{2}[0,2], I_{2}[0,3], I_{2}[0,4], I_{2}[1,2], I_{2}[1,3], I_{2}[1,4], I_{2}[2,3], I_{2}[2,4], I_{2}[3,4]$,

Triangles: $I_{3}[0,1,2], I_{3}[0,1,3], I_{3}[0,1,4], I_{3}[0,2,3], I_{3}[0,2,4], I_{3}[0,3,4], I_{3}[1,2,3], I_{3}[1,2,4], I_{3}[1,3,4], I_{3}[2,3,4]$,

Box: $I_{4}[0,1,2,3], I_{4}[0,2,3,4], I_{4}[0,1,2,4], I_{4}[1,2,3,4]$,

Pentagon: $I_{5}[0,1,2,3,4]$.

Then the reduction of the tensor pentagon is given by

$$
\begin{aligned}
I_{5}^{(m)}= & \sum_{i=0}^{4} C_{0}^{(i)}(m \mid 4) I_{1}[i]+\sum_{0 \leq i_{1}<i_{2} \leq 4} C^{\left(i_{1}, i_{2}\right)}(m \mid 4) I_{2}\left[i_{1}, i_{2}\right]+\sum_{0 \leq i_{1}<i_{2}<i_{3} \leq 4} C^{\left(i_{1}, i_{2}, i_{3}\right)}(m \mid 4) I_{3}\left[i_{1}, i_{2}, i_{3}\right] \\
& +\sum_{0 \leq i_{1}<i_{2}<i_{3}<i_{4} \leq 4} C^{\left(i_{1}, i_{2}, i_{3}, i_{4}\right)}(m \mid 4) I_{4}\left[i_{1}, i_{2}, i_{3}, i_{4}\right]+C^{(0,1,2,3,4)}(m \mid 4) I_{5}[0,1,2,3,4] .
\end{aligned}
$$

(a) $m=1$

Reduction coefficients of tadpoles, bubbles, triangles

All reduction coefficients vanish.

Reduction coefficients of boxes

$$
\begin{aligned}
C^{(0,1,2,3)}(1 \mid 4)= & \frac{s_{01} G(2,3,4 ; 1,2,3)-s_{02} G(1,3,4 ; 1,2,3)+s_{03} G(1,2,4 ; 1,2,3)}{G(1,2,3,4 ; 1,2,3,4)} \\
& -\frac{s_{04} G(1,2,3)}{G(1,2,3,4 ; 1,2,3,4)} .
\end{aligned}
$$

Choosing $j_{0}=4, j_{1}=1, j_{2}=2, j_{3}=3$ in (3.16), we have

$$
\begin{aligned}
C^{(1,2,3,4)}(1 \mid 4)= & \left.C^{(0,1,2,3)}(1 \mid 4)\right|_{K_{1} \rightarrow K_{1}-K_{4}, K_{2} \rightarrow K_{2}-K_{4}, K_{3} \rightarrow K_{3}-K_{4}, K_{4} \rightarrow-K_{4}, M_{0} \leftrightarrow M_{4}} \\
= & \frac{\left(-s_{01}+s_{04}\right) G\left(K_{2}-K_{4}, K_{3}-K_{4}, K_{4} ; K_{1}-K_{4}, K_{2}-K_{4}, K_{3}-K_{4}\right)}{G\left(K_{1}-K_{4}, K_{2}-K_{4}, K_{3}-K_{4}, K_{4} ; K_{1}-K_{4}, K_{2}-K_{4}, K_{3}-K_{4}, K_{4}\right)} \\
& +\frac{\left(s_{02}-s_{04}\right) G\left(K_{1}-K_{4}, K_{3}-K_{4}, K_{4} ; K_{1}-K_{4}, K_{2}-K_{4}, K_{3}-K_{4}\right)}{G\left(K_{1}-K_{4}, K_{2}-K_{4}, K_{3}-K_{4}, K_{4} ; K_{1}-K_{4}, K_{2}-K_{4}, K_{3}-K_{4}, K_{4}\right)} \\
& -\frac{\left(s_{03}-s_{04}\right) G\left(K_{1}-K_{4}, K_{2}-K_{4}, K_{4} ; K_{1}-K_{4}, K_{2}-K_{4}, K_{3}-K_{4}\right)}{G\left(K_{1}-K_{4}, K_{2}-K_{4}, K_{3}-K_{4}, K_{4} ; K_{1}-K_{4}, K_{2}-K_{4}, K_{3}-K_{4}, K_{4}\right)} \\
& +\frac{s_{04} G\left(K_{1}-K_{4}, K_{2}-K_{4}, K_{3}-K_{4} ; K_{1}-K_{4}, K_{2}-K_{4}, K_{3}-K_{4}\right)}{G\left(K_{1}-K_{4}, K_{2}-K_{4}, K_{3}-K_{4}, K_{4} ; K_{1}-K_{4}, K_{2}-K_{4}, K_{3}-K_{4}, K_{4}\right)} .
\end{aligned}
$$

Reduction coefficients of pentagon

$$
C^{(0,1,2,3,4)}(1 \mid 4)=c_{1,0,0,0}^{(0,1,2,3,4)}(1) s_{01}+c_{0,1,0,0}^{(0,1,2,3,4)}(1) s_{02}+c_{0,0,1,0}^{(0,1,2,3,4)}(1) s_{03}+c_{0,0,0,1}^{(0,1,2,3,4)}(1) s_{04},
$$


where

$$
\begin{gathered}
c_{1,0,0,0}^{(0,1,2,3,4)}(1)=\frac{f_{1} G(2,3,4)-f_{2} G(2,3,4 ; 1,3,4)+f_{3} G(2,3,4 ; 1,2,4)}{G(1,2,3,4 ; 1,2,3,4)}-\frac{f_{4} G(2,3,4 ; 1,2,3)}{G(1,2,3,4 ; 1,2,3,4)}, \\
c_{0,1,0,0}^{(0,1,2,3,4)}(1)=\left.c_{1,0,0,0}^{(0,1,2,3,4)}(1)\right|_{1 \leftrightarrow 2}, \\
c_{0,0,1,0}^{(0,1,2,3,4)}(1)=\left.c_{1,0,0,0}^{(0,1,2,3,4)}(1)\right|_{1 \leftrightarrow 3}, \\
c_{0,0,0,1}^{(0,1,2,3,4)}(1)=\left.c_{1,0,0,0}^{(0,1,2,3,4)}(1)\right|_{1 \leftrightarrow 4} .
\end{gathered}
$$

(b) $m=2$

Reduction coefficients of tadpoles, bubbles

All reduction coefficients vanish.

Reduction coefficients of triangles

$$
\begin{aligned}
C^{(0,1,2)(2 \mid 4)=} & -\frac{s_{04}^{2} G(1,2 ; 1,2) G(1,2,4 ; 1,2,3)}{G(1,2,4 ; 1,2,4) G(1,2,3,4 ; 1,2,3,4)}-\frac{s_{03}^{2} G(1,2 ; 1,2) G(1,2,4 ; 1,2,3)}{G(1,2,3) G(1,2,3,4 ; 1,2,3,4)} \\
& -\frac{s_{01} G(2,3,4 ; 1,2,3)\left(s_{01} G(2,3 ; 1,2)-2 s_{02} G(1,3 ; 1,2)+2 s_{03} G(1,2 ; 1,2)\right)}{G(1,2,3) G(1,2,3,4 ; 1,2,3,4)} \\
& +\frac{s_{02} G(1,3,4 ; 1,2,3)\left(2 s_{03} G(1,2 ; 1,2)-s_{02} G(1,3 ; 1,2)\right)}{G(1,2,3) G(1,2,3,4 ; 1,2,3,4)} \\
& +\frac{s_{01}^{2} G(2,4 ; 1,2) G(2,3,4 ; 1,2,4)}{G(1,2,4 ; 1,2,4) G(1,2,3,4 ; 1,2,3,4)} \\
& +\frac{s_{02} G(1,4 ; 1,2)\left(s_{02} G(1,3,4 ; 1,2,4)-2 s_{01} G(2,3,4 ; 1,2,4)\right)}{G(1,2,4 ; 1,2,4) G(1,2,3,4 ; 1,2,3,4)} \\
& +\frac{2 s_{04} G(1,2 ; 1,2)\left(s_{01} G(2,3,4 ; 1,2,4)-s_{02} G(1,3,4 ; 1,2,4)+s_{03} G(1,2,4 ; 1,2,4)\right)}{G(1,2,4 ; 1,2,4) G(1,2,3,4 ; 1,2,3,4)}
\end{aligned}
$$

Choosing $j_{0}=3, j_{1}=1, j_{2}=2$ in (3.16), we have

$$
C^{(1,2,3)}(2 \mid 4)=\left.\left(C^{(0,1,2)}(2 \mid 4)\right)\right|_{K_{i} \rightarrow K_{i}-K_{3}, i \neq 3 ; K_{3} \rightarrow-K_{3}, M_{0} \leftrightarrow M_{3}} .
$$

Reduction coefficients of boxes

$$
\begin{aligned}
C^{(0,1,2,3)}(2 \mid 4)= & \frac{1}{M_{0}^{2}}\left[c_{0,0,0,0}^{(0,1,2,3)}(2) M_{0}^{2} s_{00}+c_{2,0,0,0}^{(0,1,2,3)}(2) s_{01}^{2}+c_{0,2,0,0}^{(0,1,2,3)}(2) s_{02}^{2}+c_{0,0,2,0}^{(0,1,2,3)}(2) s_{03}^{2}\right. \\
& +c_{0,0,0,2}^{(0,1,2,3)}(2) s_{04}^{2}+c_{1,1,0,0}^{(0,1,2,3)}(2) s_{01} s_{02}+c_{0,1,1,0}^{(0,1,2,3)}(2) s_{02} s_{03}+c_{1,0,1,0}^{(0,1,2,3)}(2) s_{01} s_{03} \\
& \left.+c_{1,0,0,1}^{(0,1,2,3)}(2) s_{01} s_{04}+c_{0,1,0,1}^{(0,1,2,3)}(2) s_{02} s_{04}+c_{0,0,1,1}^{(0,1,2,3)}(2) s_{03} s_{04}\right]
\end{aligned}
$$

where 


$$
\begin{aligned}
& c_{2,0,0,0}^{(0,1,2,3)}(2)=\frac{(D-3) f_{1} M_{0}^{2} G(2,3,4 ; 1,2,3) G(2,3,4)}{(D-4) G(1,2,3,4 ; 1,2,3,4)^{2}}-\frac{M_{0}^{2} G(2,3,4)\left(f_{2} G(1,3,4 ; 1,2,3)-f_{3} G(1,2,4 ; 1,2,3)\right)}{(D-4) G(1,2,3,4 ; 1,2,3,4)^{2}} \\
& -\frac{f_{4} M_{0}^{2} G(1,2,3) G(2,3,4)}{(D-4) G(1,2,3,4 ; 1,2,3,4)^{2}}-\frac{f_{4} M_{0}^{2} G(2,3,4 ; 1,2,3)^{2}}{G(1,2,3,4 ; 1,2,3,4)^{2}} \\
& +G(2,3,4 ; 1,2,3) \frac{M_{0}^{2}\left(f_{1} G(2,3 ; 2,3)-f_{2} G(2,3 ; 1,3)+f_{3} G(2,3 ; 1,2)\right)}{G(1,2,3) G(1,2,3,4 ; 1,2,3,4)} \\
& -G(2,3,4 ; 1,2,3) \frac{M_{0}^{2}\left(f_{2} G(2,3,4 ; 1,3,4)-f_{3} G(2,3,4 ; 1,2,4)\right)}{G(1,2,3,4 ; 1,2,3,4)^{2}}, \\
& c_{0,0,0,2}^{(0,1,2,3)}(2)=\frac{f_{1}(G(2,3,4 ; 1,2,3)}{(4-D) G(1,2,3,4)}+\frac{f_{2} G(1,3,4 ; 1,2,3)}{(D-4) G(1,2,3,4)}+\frac{f_{3} G(1,2,4 ; 1,2,3)}{(4-D) G(1,2,3,4)}+\frac{f_{4} G(1,2,3)}{(D-4) G(1,2,3,4)}, \\
& c_{1,1,0,0}^{(0,1,2,3)}(2)=\frac{2(D-3) f_{2} M_{0}^{2} G(1,3,4 ; 1,2,3) G(2,3,4 ; 1,3,4)}{(D-4) G(1,2,3,4 ; 1,2,3,4)^{2}} \\
& +\frac{2 M_{0}^{2} G(2,3,4 ; 1,3,4)}{(D-4) G(1,2,3,4 ; 1,2,3,4)^{2}}\left[f_{4} G(1,2,3)-f_{3} G(1,2,4 ; 1,2,3)\right] \\
& +\frac{2 M_{0}^{2} G(2,3,4 ; 1,2,3)}{G(1,2,3,4 ; 1,2,3,4)^{2}}\left[f_{4} G(1,3,4 ; 1,2,3)-\frac{f_{1} G(2,3,4 ; 1,3,4)}{(D-4)}\right] \\
& -\frac{2 M_{0}^{2} G(1,3,4 ; 1,2,3)}{G(1,2,3,4 ; 1,2,3,4)^{2}}\left[f_{1} G(2,3,4)-f_{3} G(2,3,4 ; 1,2,4)\right] \\
& -\frac{2 M_{0}^{2} G(2,3,4 ; 1,2,3)\left(f_{1} G(2,3 ; 1,3)-f_{2} G(1,3 ; 1,3)+f_{3} G(1,3 ; 1,2)\right)}{G(1,2,3) G(1,2,3,4 ; 1,2,3,4)}, \\
& c_{0,0,0,2}^{(0,1,2)}(2)=\frac{(D-3) M_{0}^{2} G(1,2,3)}{(D-4) G(1,2,3,4 ; 1,2,3,4)^{2}}\left[f_{1}(G(2,3,4 ; 1,2,3))\right. \\
& \left.-f_{2} G(1,3,4 ; 1,2,3)+f_{3} G(1,2,4 ; 1,2,3)-f_{4} G(1,2,3)\right] \text {, } \\
& c_{1,0,0,1}^{(0,1,2,3)}(2)=-\frac{2 f_{1} M_{0}^{2} G(2,3,4 ; 1,2,3)^{2}}{(D-4) G(1,2,3,4 ; 1,2,3,4)^{2}} \\
& +\frac{2 M_{0}^{2} G(2,3,4 ; 1,2,3)}{(D-4) G(1,2,3,4 ; 1,2,3,4)^{2}}\left[f_{2} G(1,3,4 ; 1,2,3)-f_{3} G(1,2,4 ; 1,2,3)\right] \\
& +\frac{2 M_{0}^{2} G(1,2,3)}{G(1,2,3,4 ; 1,2,3,4)^{2}}\left[\frac{(D-3) f_{4} G(2,3,4 ; 1,2,3)}{(D-4)}\right. \\
& \left.-f_{1} G(2,3,4)+f_{2} G(2,3,4 ; 1,3,4)-f_{3} G(2,3,4 ; 1,2,4)\right] \text {. }
\end{aligned}
$$

Other expansion coefficients can be obtained by using the permutation symmetry,

$$
\begin{aligned}
& c_{0,2,0,0}^{(0,1,2,3)}(2)=\left.c_{2,0,0,0}^{(0,1,2,3)}(2)\right|_{1 \leftrightarrow 2}, \\
& c_{0,0,2,0}^{(0,1,2,3)}(2)=\left.c_{2,0,0,0}^{(0,1,2,3)}(2)\right|_{1 \leftrightarrow 3}, \\
& c_{1,0,1,0}^{(0,1,2,3)}(2)=\left.c_{1,1,0,0}^{(0,1,2,3)}(2)\right|_{2 \leftrightarrow 3}, \\
& c_{0,1,1,0}^{(0,1,2)}(2)=\left.c_{1,1,0,0}^{(0,1,2,3)}(2)\right|_{1 \leftrightarrow 2}, \\
& c_{0,1,0,1}^{(0,1,2)}(2)=\left.c_{1,0,0,1}^{(0,1,2,3)}(2)\right|_{1 \leftrightarrow 2}, \\
& c_{0,0,1,1}^{(0,1,2,3)}(2)=\left.c_{1,0,0,1}^{(0,1,2,3)}(2)\right|_{1 \leftrightarrow 3} .
\end{aligned}
$$


Choosing $j_{0}=4, j_{1}=1, j_{2}=2, j_{3}=3$ in (3.16), we have

$$
\begin{aligned}
C^{(1,2,3,4)}(2 \mid 4)= & \left.4 s_{04}\left(C^{(0,1,2,3)}(1 \mid 4)\right)\right|_{K_{i} \rightarrow K_{i}-K_{4}, i<4 ; K_{4} \rightarrow-K_{4}, M_{0} \leftrightarrow M_{4}} \\
& +\left.\left(C^{(0,1,2,3)}(2 \mid 4)\right)\right|_{K_{i} \rightarrow K_{i}-K_{4}, i<4 ; K_{4} \rightarrow-K_{4}, M_{0} \leftrightarrow M_{4}} .
\end{aligned}
$$

The reduction coefficient of pentagon

$$
\begin{aligned}
C^{(0,1,2,3,4)}(2 \mid 4)= & {\left[c_{0,0,0,0}^{(0,1,2,3,4)}(2) M_{0}^{2} s_{00}+c_{2,0,0,0}^{(0,1,2,3,4)}(2) s_{01}^{2}+c_{0,2,0,0}^{(0,1,2,3,4)}(2) s_{02}^{2}+c_{0,0,2,0}^{(0,1,2,3,4)}(2) s_{03}^{2}\right.} \\
& +c_{0,0,0,2}^{(0,1,2,3,4)}(2) s_{04}^{2}+c_{1,1,0,0}^{(0,1,2,3,4)}(2) s_{01} s_{02}+c_{0,1,1,0}^{(0,1,2,3)}(2) s_{02} s_{03}+c_{1,0,1,0}^{(0,1,2,3,4)}(2) s_{01} s_{03} \\
& \left.+c_{1,0,0,1}^{(0,1,2,3,4)}(2) s_{01} s_{04}+c_{0,1,0,1}^{(0,1,2,3,4)}(2) s_{02} s_{04}+c_{0,0,1,1}^{(0,1,2,3,4)}(2) s_{03} s_{04}\right] .
\end{aligned}
$$

where

$$
\begin{aligned}
& c_{0,0,0,0}^{(0,1,2,3,4)}(2)=-\frac{f_{1}^{2} G(2,3,4)}{(D-4) M_{0}^{2} G(1,2,3,4 ; 1,2,3,4)}+\frac{2 f_{2} f_{1} G(2,3,4 ; 1,3,4)}{(D-4) M_{0}^{2} G(1,2,3,4 ; 1,2,3,4)} \\
& -\frac{2 f_{3} f_{1} G(2,3,4 ; 1,2,4)}{(D-4) M_{0}^{2} G(1,2,3,4 ; 1,2,3,4)}+\frac{2 f_{4} f_{1} G(2,3,4 ; 1,2,3)}{(D-4) M_{0}^{2} G(1,2,3,4 ; 1,2,3,4)} \\
& -\frac{f_{2}^{2} G(1,3,4)}{(D-4) M_{0}^{2} G(1,2,3,4 ; 1,2,3,4)}-\frac{f_{3}^{2} G(1,2,4)}{(D-4) M_{0}^{2} G(1,2,3,4 ; 1,2,3,4)} \\
& -\frac{f_{4}^{2} G(1,2,3)}{(D-4) M_{0}^{2} G(1,2,3,4 ; 1,2,3,4)}+\frac{2 f_{2} f_{3} G(1,3,4 ; 1,2,4)}{(D-4) M_{0}^{2} G(1,2,3,4 ; 1,2,3,4)} \\
& -\frac{2 f_{2} f_{4} G(1,3,4 ; 1,2,3)}{(D-4) M_{0}^{2} G(1,2,3,4 ; 1,2,3,4)}+\frac{2 f_{3} f_{4} G(1,2,4 ; 1,2,3)}{(D-4) M_{0}^{2} G(1,2,3,4 ; 1,2,3,4)} \\
& +\frac{4}{D-4} \\
& c_{2,0,0,0}^{(0,1,2,3,4)}(2)=\frac{(D-3) f_{1}^{2} G(2,3,4)^{2}}{(D-4) G(1,2,3,4 ; 1,2,3,4)^{2}}-\frac{2(D-3) f_{2} f_{1} G(2,3,4 ; 1,3,4) G(2,3,4)}{(D-4) G(1,2,3,4 ; 1,2,3,4)^{2}} \\
& +\frac{2(D-3) f_{3} f_{1} G(2,3,4 ; 1,2,4) G(2,3,4)}{(D-4) G(1,2,3,4 ; 1,2,3,4)^{2}}-\frac{2(D-3) f_{4} f_{1} G(2,3,4 ; 1,2,3) G(2,3,4)}{(D-4) G(1,2,3,4 ; 1,2,3,4)^{2}} \\
& +f_{2}^{2}\left(\frac{G(1,3,4) G(2,3,4)}{(D-4) G(1,2,3,4 ; 1,2,3,4)^{2}}+\frac{G(2,3,4 ; 1,3,4)^{2}}{G(1,2,3,4 ; 1,2,3,4)^{2}}\right) \\
& +\frac{f_{3}^{2}\left((D-4) G(2,3,4 ; 1,2,4)^{2}+G(1,2,4 ; 1,2,4) G(2,3,4)\right)}{(D-4) G(1,2,3,4 ; 1,2,3,4)^{2}} \\
& +f_{4}^{2}\left(\frac{G(1,2,3) G(2,3,4)}{(D-4) G(1,2,3,4 ; 1,2,3,4)^{2}}+\frac{G(2,3,4 ; 1,2,3)^{2}}{G(1,2,3,4 ; 1,2,3,4)^{2}}\right) \\
& +f_{2} f_{3}\left(-\frac{2 G(1,3,4 ; 1,2,4) G(2,3,4)}{(D-4) G(1,2,3,4 ; 1,2,3,4)^{2}}-\frac{2 G(2,3,4 ; 1,2,4) G(2,3,4 ; 1,3,4)}{G(1,2,3,4 ; 1,2,3,4)^{2}}\right) \\
& +f_{2} f_{4}\left(\frac{2 G(1,3,4 ; 1,2,3) G(2,3,4)}{(D-4) G(1,2,3,4 ; 1,2,3,4)^{2}}+\frac{2 G(2,3,4 ; 1,2,3) G(2,3,4 ; 1,3,4)}{G(1,2,3,4 ; 1,2,3,4)^{2}}\right) \\
& +f_{3} f_{4}\left(-\frac{2 G(1,2,4 ; 1,2,3) G(2,3,4)}{(D-4) G(1,2,3,4 ; 1,2,3,4)^{2}}-\frac{2 G(2,3,4 ; 1,2,3) G(2,3,4 ; 1,2,4)}{G(1,2,3,4 ; 1,2,3,4)^{2}}\right) \\
& 4 M_{0}^{2} G(2,3,4) \\
& -\overline{(D-4) G(1,2,3,4 ; 1,2,3,4)} \text {, }
\end{aligned}
$$




$$
\begin{aligned}
& c_{1,1,0,0}^{(0,1,2,3,4)}(2)=-\frac{2(D-3) G(2,3,4 ; 1,3,4) G(2,3,4) f_{1}^{2}}{(D-4) G(1,2,3,4 ; 1,2,3,4)^{2}}+\frac{8 G(2,3,4 ; 1,3,4) M_{0}^{2}}{(D-4) G(1,2,3,4 ; 1,2,3,4)} \\
& +\left(\frac{2(D-2) G(2,3,4 ; 1,3,4)^{2}}{(D-4) G(1,2,3,4 ; 1,2,3,4)^{2}}+\frac{2 G(1,3,4) G(2,3,4)}{G(1,2,3,4 ; 1,2,3,4)^{2}}\right) f_{2} f_{1} \\
& +\left(-\frac{2(D-2) G(2,3,4 ; 1,2,4) G(2,3,4 ; 1,3,4)}{(D-4) G(1,2,3,4 ; 1,2,3,4)^{2}}-\frac{2 G(1,3,4 ; 1,2,4) G(2,3,4)}{G(1,2,3,4 ; 1,2,3,4)^{2}}\right) f_{3} f_{1} \\
& +\left(\frac{2(D-2) G(2,3,4 ; 1,2,3) G(2,3,4 ; 1,3,4)}{(D-4) G(1,2,3,4 ; 1,2,3,4)^{2}}+\frac{2 G(1,3,4 ; 1,2,3) G(2,3,4)}{G(1,2,3,4 ; 1,2,3,4)^{2}}\right) f_{4} f_{1} \\
& +\left(-\frac{2 G(1,3,4 ; 1,2,4) G(2,3,4 ; 1,2,4)}{G(1,2,3,4 ; 1,2,3,4)^{2}}-\frac{2 G(1,2,4 ; 1,2,4) G(2,3,4 ; 1,3,4)}{(D-4) G(1,2,3,4 ; 1,2,3,4)^{2}}\right) f_{3}^{2} \\
& +\left(-\frac{2 G(1,3,4 ; 1,2,3) G(2,3,4 ; 1,2,3)}{G(1,2,3,4 ; 1,2,3,4)^{2}}-\frac{2 G(1,2,3) G(2,3,4 ; 1,3,4)}{(D-4) G(1,2,3,4 ; 1,2,3,4)^{2}}\right) f_{4}^{2} \\
& +\left(\frac{2 G(1,3,4) G(2,3,4 ; 1,2,4)}{G(1,2,3,4 ; 1,2,3,4)^{2}}+\frac{2(D-2) G(1,3,4 ; 1,2,4) G(2,3,4 ; 1,3,4)}{(D-4) G(1,2,3,4 ; 1,2,3,4)^{2}}\right) f_{2} f_{3} \\
& +\left(-\frac{2 G(1,3,4) G(2,3,4 ; 1,2,3)}{G(1,2,3,4 ; 1,2,3,4)^{2}}-\frac{2(D-2) G(1,3,4 ; 1,2,3) G(2,3,4 ; 1,3,4)}{(D-4) G(1,2,3,4 ; 1,2,3,4)^{2}}\right) f_{2} f_{4} \\
& +\left(\frac{2 G(1,3,4 ; 1,2,4) G(2,3,4 ; 1,2,3)}{G(1,2,3,4 ; 1,2,3,4)^{2}}+\frac{2 G(1,3,4 ; 1,2,3) G(2,3,4 ; 1,2,4)}{G(1,2,3,4 ; 1,2,3,4)^{2}}\right) f_{3} f_{4} \\
& +\frac{4 G(1,2,4 ; 1,2,3) G(2,3,4 ; 1,3,4)}{(D-4) G(1,2,3,4 ; 1,2,3,4)^{2}} f_{3} f_{4}-\frac{2(D-3) G(1,3,4) G(2,3,4 ; 1,3,4) f_{2}^{2}}{(D-4) G(1,2,3,4 ; 1,2,3,4)^{2}} \text {. }
\end{aligned}
$$

Other expansion coefficients can be got by using permutation symmetry,

$$
\begin{aligned}
& c_{0,2,0,0}^{(0,1,2,3,4)}(2)=\left.c_{2,0,0,0}^{(0,1,2,3,4)}(2)\right|_{1 \leftrightarrow 2}, \\
& c_{0,0,2,0}^{(0,1,2,3,4)}(2)=\left.c_{2,0,0,0}^{(0,1,2,3,4)}(2)\right|_{1 \leftrightarrow 3}, \\
& c_{0,0,0,2}^{(0,1,2,3,4)}(2)=\left.c_{2,0,0,0}^{(0,1,2,3,4)}(2)\right|_{1 \leftrightarrow 4}, \\
& c_{1,0,1,0}^{(0,1,2,3,4)}(2)=\left.c_{1,1,0,0}^{(0,1,2,3,4)}(2)\right|_{2 \leftrightarrow 3}, \\
& c_{0,1,1,0}^{(0,1,2,3,4)}(2)=\left.c_{1,1,0,0}^{(0,1,2,3,4)}(2)\right|_{1 \leftrightarrow 2}, \\
& c_{0,1,0,1}^{(0,1,2,3,4)}(2)=\left.c_{1,0,0,1}^{(0,1,2,3,4)}(2)\right|_{1 \leftrightarrow 2}, \\
& c_{0,0,1,1}^{(0,1,2,3,4)}(2)=\left.c_{1,0,0,1}^{(0,1,2,3,4)}(2)\right|_{1 \leftrightarrow 3}, \\
& c_{1,0,0,1}^{(0,1,2,3,4)}(2)=\left.c_{1,0,0,1}^{(0,1,2,3,4)}(2)\right|_{1 \leftrightarrow 4} .
\end{aligned}
$$

[1] L. M. Brown and R. P. Feynman, Radiative corrections to Compton scattering, Phys. Rev. 85, 231 (1952).

[2] D. B. Melrose, Reduction of Feynman diagrams, Nuovo Cimento 40, 181 (1965).

[3] G. Passarino and M. J. G. Veltman, One loop corrections for $\mathrm{e}+\mathrm{e}-$ annihilation into $\mathrm{mu}+\mathrm{mu}-$ in the Weinberg model, Nucl. Phys. B160, 151 (1979).
[4] G. 't Hooft and M. J. G. Veltman, Scalar one loop integrals, Nucl. Phys. B153, 365 (1979).

[5] W. L. van Neerven and J. A. M. Vermaseren, Large loop integrals, Phys. Lett. 137B, 241 (1984).

[6] R. G. Stuart, Algebraic reduction of one loop Feynman diagrams to scalar integrals, Comput. Phys. Commun. 48, 367 (1988). 
[7] G. J. van Oldenborgh and J. A. M. Vermaseren, New algorithms for one loop integrals, Z. Phys. C 46, 425 (1990).

[8] Z. Bern, L. J. Dixon, and D. A. Kosower, Dimensionally regulated one loop integrals, Phys. Lett. B 302, 299 (1993); 318, 649(E) (1993).

[9] Z. Bern, L. J. Dixon, and D. A. Kosower, Dimensionally regulated pentagon integrals, Nucl. Phys. B412, 751 (1994).

[10] J. Fleischer, F. Jegerlehner, and O. V. Tarasov, Algebraic reduction of one loop Feynman graph amplitudes, Nucl. Phys. B566, 423 (2000).

[11] T. Binoth, J. P. Guillet, and G. Heinrich, Reduction formalism for dimensionally regulated one loop $\mathrm{N}$ point integrals, Nucl. Phys. B572, 361 (2000).

[12] A. Denner and S. Dittmaier, Reduction of one loop tensor five point integrals, Nucl. Phys. B658, 175 (2003).

[13] G. Duplancic and B. Nizic, Reduction method for dimensionally regulated one loop $\mathrm{N}$ point Feynman integrals, Eur. Phys. J. C 35, 105 (2004).

[14] A. Denner and S. Dittmaier, Reduction schemes for oneloop tensor integrals, Nucl. Phys. B734, 62 (2006).

[15] R. K. Ellis and G. Zanderighi, Scalar one-loop integrals for QCD, J. High Energy Phys. 02 (2008) 002.

[16] G. Ossola, C. G. Papadopoulos, and R. Pittau, Reducing full one-loop amplitudes to scalar integrals at the integrand level, Nucl. Phys. B763, 147 (2007).

[17] Z. Bern, L. J. Dixon, D. C. Dunbar, and D. A. Kosower, Fusing gauge theory tree amplitudes into loop amplitudes, Nucl. Phys. B435, 59 (1995).

[18] K. G. Chetyrkin and F. V. Tkachov, Integration by parts: The algorithm to calculate beta functions in 4 loops, Nucl. Phys. B192, 159 (1981).

[19] F. V. Tkachov, A theorem on analytical calculability of four loop renormalization group functions, Phys. Lett. 100B, 65 (1981).

[20] G. Ossola, C. G. Papadopoulos, and R. Pittau, Reducing full one-loop amplitudes to scalar integrals at the integrand level, Nucl. Phys. B763, 147 (2007).

[21] G. Ossola, C. G. Papadopoulos, and R. Pittau, Numerical evaluation of six-photon amplitudes, J. High Energy Phys. 07 (2007) 085.

[22] R. K. Ellis, W. T. Giele, and Z. Kunszt, A numerical unitarity formalism for evaluating one-loop amplitudes, J. High Energy Phys. 03 (2008) 003.

[23] Z. Bern, L. J. Dixon, D. C. Dunbar, and D. A. Kosower, One loop $\mathrm{n}$ point gauge theory amplitudes, unitarity and collinear limits, Nucl. Phys. B425, 217 (1994).

[24] R. Britto, F. Cachazo, and B. Feng, Generalized unitarity and one-loop amplitudes in $N=4$ super-Yang-Mills, Nucl. Phys. B725, 275 (2005).
[25] R. Britto, E. Buchbinder, F. Cachazo, and B. Feng, Oneloop amplitudes of gluons in SQCD, Phys. Rev. D 72, 065012 (2005).

[26] J. M. Campbell, E. W. N. Glover, and D. J. Miller, One loop tensor integrals in dimensional regularization, Nucl. Phys. B498, 397 (1997).

[27] Z. Bern, L. J. Dixon, and D. A. Kosower, One loop amplitudes for $\mathrm{e}+\mathrm{e}-$ to four partons, Nucl. Phys. B513, 3 (1998).

[28] C. Anastasiou, R. Britto, B. Feng, Z. Kunszt, and P. Mastrolia, Unitarity cuts and Reduction to master integrals in d dimensions for one-loop amplitudes, J. High Energy Phys. 03 (2007) 111.

[29] R. Britto and E. Mirabella, Single cut integration, J. High Energy Phys. 01 (2011) 135.

[30] C. Anastasiou, R. Britto, B. Feng, Z. Kunszt, and P. Mastrolia, D-dimensional unitarity cut method, Phys. Lett. B 645, 213 (2007).

[31] R. Britto and B. Feng, Unitarity cuts with massive propagators and algebraic expressions for coefficients, Phys. Rev. D 75, 105006 (2007).

[32] R. Britto and B. Feng, Integral coefficients for one-loop amplitudes, J. High Energy Phys. 02 (2008) 095.

[33] R. Britto, B. Feng, and P. Mastrolia, Closed-form decomposition of one-loop massive amplitudes, Phys. Rev. D 78, 025031 (2008).

[34] R. Britto, B. Feng, and G. Yang, Polynomial structures in one-loop amplitudes, J. High Energy Phys. 09 (2008) 089.

[35] B. Feng and H. Wang, Analytic structure of one-loop coefficients, J. High Energy Phys. 05 (2013) 104.

[36] B. Feng, T. Li, and X. Li, Analytic tadpole coefficients of one-loop integrals, J. High Energy Phys. 09 (2021) 081.

[37] R. Britto, F. Cachazo, and B. Feng, New recursion relations for tree amplitudes of gluons, Nucl. Phys. B715, 499 (2005).

[38] R. Britto, F. Cachazo, B. Feng, and E. Witten, Direct Proof of Tree-Level Recursion Relation in Yang-Mills Theory, Phys. Rev. Lett. 94, 181602 (2005).

[39] A. V. Smirnov, Algorithm FIRE—Feynman integral REduction, J. High Energy Phys. 10 (2008) 107.

[40] A. V. Smirnov, FIRE5: A C ++ implementation of Feynman integral REduction, Comput. Phys. Commun. 189, 182 (2015).

[41] A. V. Smirnov and V.A. Smirnov, FIRE4, LiteRed and accompanying tools to solve integration by parts relations, Comput. Phys. Commun. 184, 2820 (2013).

[42] A. V. Smirnov and F. S. Chuharev, FIRE6: Feynman integral REduction with modular arithmetic, Comput. Phys. Commun. 247, 106877 (2020).

[43] R. N. Lee, LiteRed 1.4: A powerful tool for reduction of multiloop integrals, J. Phys. Conf. Ser. 523, 012059 (2014). 